\title{
Die Selbstunterscheidungen der Moderne
}

\author{
Klaus Lichtblau \\ Die Selbstunterscheidungen der Moderne[1]
}

"Die Autorität des Neuen ist die des geschichtlich Unausweichlichen. Insofern impliziert es objektiv Kritik am Individuum, seinem Vehikel: ästhetisch schürzt im Neuen sich der Knoten von Individuum und Gesellschaft. Die Erfahrung der Moderne sagt mehr, obwohl ihr Begriff, wie immer auch qualitativ, an seiner Abstraktheit laboriert. Er ist privativ, von Anbeginn mehr Negation dessen, was nun nicht mehr sein soll, als positive Parole. Er negiert aber nicht, wie von je die Stile, vorhergehende Kunstübungen, sondern Tradition als solche; insofern ratifiziert er erst das bürgerliche Prinzip in der Kunst."

Theodor W. Adorno

Die Moderne scheint mittlerweile selbst zunehmend ins Alter gekommen zu sein. Zumindest häufen sich seit einiger Zeit nicht nur im Fach Soziologie vermehrt diejenigen Stimmen, die ihr entweder einen tiefgreifenden Gestaltwandel oder sogar ihre eigene historische Überlebtheit zu bescheinigen versuchen. Eine solche nostalgische Stimmung hat offensichtlich immer dann Konjunktur, wenn einschneidende Veränderungen in der politischen und sozialen Arena sowie der damit verbundene Wandel des Zeitgeistes auf einen möglichen Kontinuitätsbruch innerhalb der Geschichte der Moderne verweisen, der noch nicht mit präzisen wissenschaftlichen Formen der Begriffsbildung beschrieben werden kann und infolgedessen ideale Rahmenbedingungen für die publizistische Breitenwirkung einer kaum mehr überschaubaren Zahl von selbsternannten Zeitdiagnostikern bietet, die sich innerhalb einer breiteren Öffentlichkeit nun selbst wechselseitig die Deutungskompetenz bezüglich des unterstellten sozialen und kulturellen Wandels zu- bzw. absprechen. Unterschiedliche disziplinäre Zugangsweisen bezüglich der Erfassung des vermeintlichen "Geistes der Zeit" konkurrieren dabei ebenso miteinander wie verschiedene generationsspezifische Erfahrungsräume und Erwartungshorizonte, die nur unter gewaltsamer Preisgabe dieser disziplinären bzw. altersspezifischen Differenzen zugunsten einer neuen übergreifenden Situationsbeschreibung aufhebbar erscheinen.[2] Und eine solche neue einheitliche Gesamtcodierung der zeitgenössischen wissenschaftlichen, kulturellen und weltanschaulichen Semantik scheitert nicht zuletzt allein schon aufgrund des grundsätzlich pluralistischen bzw. dezentrischen Charakters moderner Gesellschaften, der eben sehr viele und zum Teil auch recht heterogene Beobachterstandpunkte zuläßt, die sich nicht mehr ohne weiteres auf einen gemeinsamen Nenner bringen lassen. Diese spezifisch "moderne" Konstellation findet aber auch ihren fachspezifischen Niederschlag in dem derzeitigen intellektuellen Zustand einer akademischen Disziplin wie der modernen Soziologie, die sich zwar von Anfang an eine spezifische zeitdiagnostische Kompetenz anmaß und dabei in Abgrenzung zu den mehr historisch orientierten Geistes- und Kulturwissenschaften der Jahrhundertwende selbst den Status einer genuinen Gegenwartswissenschaft zusprach, die aber offensichtlich heute nicht einmal mehr in der Lage ist, die Vielzahl der von ihr selbst in die Welt gesetzten theoretischen Differenzen und grundbegrifflichen Unterscheidungen zugunsten einer verbindlicheren Deutung der modernen Welt auf der Grundlage eines einheitlichen disziplinären Kanons zu überwinden.[3]

Totgesagte leben bekanntlich meistens länger als erwartet. Dies gilt nicht zuletzt auch für jenes Wirtschaftssystem, dem nicht nur bereits Karl Marx Mitte des vorigen Jahrhunderts sein baldiges Ende voraussagte, sondern dem auch der berühmte deutsche Nationalökonom und Sozialwissenschaftler Werner Sombart unter dem Eindruck der außerordentlich erfolgreichen Organisation der deutschen Kriegswirtschaft während des Ersten Weltkrieges und des sich dabei bereits am Horizont abzeichnenden Planstaates im Unterschied zu den voraufgegangenen Epochen des Früh- und Hochkapitalismus den Charakter eines "Spätkapitalismus" zusprechen zu können meinte, der ihm zufolge nun offensichtlich bald einer neuen Mischform 
von Privat- und Staatswirtschaft im Sinne eines spezifischen "deutschen Sozialismus" Platz machen sollte.[4] Nun, wir wissen heute, daß schließlich alles ganz anders gekommen ist und daß es nicht einmal dem im Gefolge des Ersten bzw. Zweiten Weltkrieges in veschiedenen Regionen dieser Welt entstandenen "realen Sozialismus" leninistischer Prägung vergönnt war, die Todesstunde des kapitalistischen Wirtschafts- und Gesellschaftssystems zu erleben, von dem er sich doch so sehr abgrenzte und als dessen legitimes geschichtsphilosophisches Erbe er sich selbst mißverstand.

Offensichtlich ist aber mit dem Niedergang des realen Sozialismus nicht nur etwas zerbrochen, das der britischpolnische Soziologe Zygmunt Bauman unlängst als die letzte historische und dabei zugleich radikalste Erscheinungsform der europäischen Moderne bezeichnet hat, sondern auch etwas, was dem im Zeitalter der europäischen Aufklärung geborenen "Projekt der Moderne" von Anfang an in seine Geburtsurkunde geschrieben war: nämlich sein zutiefst utopischer bzw. normativer Charakter. Und dieser spezifisch utopisch-normative Gehalt kam nicht nur in jener kulturellen Antinomie zum Ausdruck, derzufolge eine Kultur, die sich selbst als eine Kampfansage gegenüber einer ihr historisch extern vorgegebenen Epoche sowie den damit verbundenen Differenzen verstand, notwendig ihre eigenen Ambivalenzen und Paradoxien heraufbeschwören muß, welche sich innerhalb einer prinzipiell pluralistisch und dezentrisch verfaßten Welt allein schon aus strukturellen Gründen ergeben müssen. Utopisch war nämlich auch der Versuch, die in dem Kollektivsingular "Moderne" zusammengefaßten verschiedenen gesellschaftlichen und kulturellen Selbstbeschreibungen im Sinne einer geschichtsphilosophischen Periodisierbarkeit auszumessen. Denn handelt es sich im vorliegenden Fall überhaupt um ein historisch konkret faßbares Zeitalter, bei dem wir ähnlich genaue bzw. ungenaue Differenzierungen vornehmen können wie bei der in der Historiographie der letzten beiden Jahrhunderte mit Vorliebe verwendeten Untergliederung der europäischen Geschichte in die alte, mittlere und neuere Geschichte oder bei der hier ebenfalls beliebten Bevorzugung der einzelnen Säkula als Maßstab für die Periodisierung der neueren Geschichte, wobei dann den einzelnen Jahrhundertwenden eine erhebliche Wahrscheinlichkeit als vermeintlichen Epochenschwellen zugesprochen werden muß?[5] Zumindest Zygmunt Bauman ist mit guten Gründen der Ansicht, daß bei dem "Projekt der Moderne" jeder Versuch einer genauen Datierung seiner Anfänge, seiner mutmaßlichen Höhepunkte sowie seines vermeintlichen Endes notwendig zum Scheitern verurteilt ist, weil sich zum einen jede Jahreszahl durch eine andere wiederlegen läßt und weil sich diese Sucht nach epochaler Einheit und Eindeutigkeit ohnedies nur um den Preis der Erzeugung der für die Moderne selbst konstitutiven Ambivalenzen und Paradoxien bzw. Pathologien befriedigen läßt. [6]

Sollte sich diese Beobachtung also weiter erhärten lassen - und es spricht aus meiner Sicht nichts dagegen, daß dies tatsächlich der Fall ist -, so bietet es sich offensichtlich an, beim Umgang mit den in diesem "Projekt der Moderne" zum Ausdruck kommenden Schwierigkeiten einer epochalen Standortbestimmung bzw. gesellschaftstheoretischen Beschreibung des gegenwärtigen Zeitalters ein anderes Verfahren anzuwenden als die von der Historiographie bevorzugte Methode der zeitlichen Datierung von Ereignissen, Strukturen und Prozessen. Nur so ist es nämlich möglich, neben der mit der Chronometrie erfaßbaren historischen Dimensionen eines Zeitalters zugleich die mit diesem "Projekt der Moderne" verbundenen spezifischen utopischen und normativen geschichtsphilosophischen Bedeutungsgehalte zu berücksichtigen. Und nur bei einer solchen alternativen Vorgehensweise ist gewährleistet, daß zugleich die unterschiedlichen disziplinären Beschreibungsmöglichkeiten des spezifischen Erfahrungsgehaltes von Modernität berücksichtigt werden, wie sie nicht nur in den philosophischen und soziologischen Diskursen, sondern eben auch zum Beispiel in den ästhetischen und literarischen Beschreibungen der Moderne zum Ausdruck kommen, die offensichtlich auf ganz andere Sachverhalte abzielen als eine vornehmlich auf die ökonomischen und sozialen Strukturen sowie den damit verbundenen normativen Kern des modernen Gesellschaftssystems bezogene theoretische Form der Gegenwartsanalyse.

Ich möchte deshalb im folgenden eine methodische Vorgehensweise vorstellen, welche in der Lage ist, vor dem Hintergrund der entsprechenden wort- und begriffsgeschichtlichen Befunde dieser disziplinären Bedeutungsvielfalt im Begriff der Moderne gerecht zu werden, ohne daß - wie zum Teil in der soziologischen Modernisierungsforschung üblich - ein seinerseit normativ aufgeladenes und insofern selbst ahistorisches bzw. 
zeitlich abgehobenes Modernitätsverständnis zur Grundlage der Beurteilung all jener anderen Modernitätsdiskurse gemacht wird, welche offensichtlich auf ganz andere Erfahrungsgehalte als die von der Soziologie wahrgenommenen abzielen. Vielleicht ist es mit einer solchen alternativen Vorgehensweise möglich, die spezifischen Beschränktheiten bzw. den "blinden Fleck" einer rein soziologischen Betrachtung der mit dem Begriff der Moderne sowie den damit im Zusammenhang stehenden weiteren begrifflichen Differenzierungsmöglichkeiten wie z.B. der zwischen Neuzeit und Moderne oder aber Postmoderne und Moderne deutlich zu machen. Ferner soll gefragt werden, welchen spezifischen Beitrag die moderne soziologische Theorie ihrerseits zu leisten vermag, um etwas mehr Klarheit in die mit dem Projekt der Moderne und seinen verschiedenen Ausuferungen einhergehende babylonische Sprachverwirrung zu bringen. Denn es ist ja nicht ausgeschlossen, daß der damit verbundene semantische Überschuß auf eine Reihe von grundlegenden begrifflichen Unterscheidungen reduziert werden kann, die selbst nicht in einem willkürlichen Verhältnis zueinander stehen, sondern deren wechselseitige Beziehung sich mit den Mitteln einer die grundbegriffliche Differenz zwischen Sozialstruktur und Semantik bzw. Gesellschaft und Kultur berücksichtigenden soziologischen Beschreibung so klären läßt, daß zugleich deutlich wird, welcher Stellenwert der modernen Soziologie selbst im Rahmen dieser Selbstunterscheidungen der Moderne zugesprochen werden muß.[7] Denn die Soziologie reflektiert ja nicht von einer Vogelperspektive aus die mit dem Begriff der Moderne aufgeworfenen zeitdiagnostischen Probleme, sondern bildet wie alle anderen Disziplinen und intellektuellen Diskurse, die sich auf das schwierige Geschäft einer epochalen Standortbestimmung der Moderne einlassen, ihrerseits nur einen partikularen Beobachterstandpunkt innerhalb jener Gesellschaft, der eben in einer Vielzahl von recht unterschiedlichen Zugangsweisen das Signum der Modernität zugesprochen wird. Insofern befindet sich die moderne Soziologie nicht nur in guter Gesellschaft, sondern bildet selbst einen konstitutiven Teil jener Kultur, die wir als die Moderne zu bezeichnen gewohnt sind. Und aus genau dem gleichen Grund bilden auch die grundbegrifflichen Unterscheidungen, welche die Soziologie selbst innerhalb dieses übergreifenden Diskurses über die Moderne einzubringen vermag, ihrerseits einen noch zu spezifizierenden Teil eben jener Unterscheidungen, mit denen das "moderne Zeitalter" bzw. genauer: die spezifischen Erfahrungsgehalte der Moderne gegenüber anders gearteten epochalen Erfahrungsweisen bzw. der auf sie bezogenen Diskurse eindeutig bezeichnet und beschrieben werden können. In genau diesem Sinne möchte ich im folgenden deshalb auch von den Selbstunterscheidungen der Moderne sprechen. Denn die "Moderne" ist ja nicht unabhängig von jenen semantischen Beschreibungen und Unterscheidungen zu denken, die ihren eigenen epochalen Erfahrungsgehalt in Abgrenzung von anderen Arten der "kulturellen Vergesellschaftung" unter Bezugnahme auf nur ihr zukommende Leitdifferenzen zu bestimmen versuchen. Und genau dieses Geschäft einer grundbegrifflichen Differenzierung der ihr zugrundeliegenden epochalen Erfahrungsgehalte ist im Falle der Moderne innerhalb des von ihr selbst vorgegebenen Bezugsrahmens offensichtlich von recht unterschiedlichen Beobachterstandpunkten aus möglich, ja geradezu zwingend geboten, um dem von ihr eröffneten historischen Erfahrungsraum und utopisch-normativen Erwartungshorizont einigermaßen gerecht werden zu können. Doch welchen Beitrag ist die Soziologie überhaupt bei diesem Geschäft einer differentiellen Bestimmung der spezifischen Erfahrungsgehalte der Moderne zu leisten in der Lage? Nun, bis vor wenigen Jahren gab es noch mehr oder weniger so etwas wie einen orthodoxen innersoziologischen Konsens darüber, was die Moderne ist bzw. als was sie nach Maßgabe der dabei in Anspruch genommenen soziologischen Tradition zu verstehen sei. Gemeint war ein auf die "klassische" grundbegriffliche Unterscheidung zwischen traditionaler und moderner Gesellschaft bezogenes analytisches Instrumentarium, welches gleichsam in einer oberlehrerhaften Weise festzulegen versuchte, was unter Zugrundelegung spezifischer gesellschaftstheoretischer Prämissen überhaupt als "modern" zu gelten habe bzw. was nicht. Die als "Moderne" gekennzeichnete Gesellschaftsformation wurde dabei als eine "Zäsur welthistorischen Ausmaßes" verstanden, deren spezifische Eigenart darin zum Ausdruck kommt, daß sie sich im Unterschied zu anderen Epochen der kulturellen Vergesellschaftung in Form einer "Selbstbegründung" bzw. "Selbstbehauptung" ohne Rückgriff auf überlieferte Traditionen gleichsam "autopoietisch" konstituiert und seither insofern auch strikt selbstbezüglich im Sinne einer operativen 
Geschlossenheit ihrer basalen Strukturen und Prozesse beständig reproduziert.[8] Zentral für diesen Operationsmodus einer nun als "Fortschritt" gedeuteten gesamtgesellschaftlichen Entwicklung ist dabei eine Form der Selbstunterscheidung der Moderne, die von fast allen klassischen und zeitgenössischen soziologischen Theorien als Prozeß einer funktionalen Differenzierung der Gesamtgesellschaft in unterschiedliche Teilsysteme beschrieben wird, der seit Simmel und Durkheim bis hin zu Talcott Parsons und Niklas Luhmann als der eigentliche sozialstrukturelle Kern von Modernität schlechthin angesehen wird. Unterstellt wird hierbei die Annahme, daß diese in sich selbst gespaltene und deshalb in einem kritischen Sinne zugleich auch "entzweite" bzw. "unversöhnte" Moderne die Freisetzung von unterschiedlichen "Wertsphären" beinhaltet, die sich nach Maßgabe jeweils teilsystemspezifischer "Leitdifferenzen" bzw. Unterscheidungen zunehmend weiter zu entfalten vermochten und deshalb nur bei Strafe des notwendigen Mißverstehens dieses funktionalen Differenzierungsprozesses mit einer vorschnellen einheitlichen Beschreibungsformel wie z.B. dem der "Rationalisierung" auf einen gemeinsamen Nenner gebracht werden können. Nicht zufällig betonte bereits Max Weber, daß der Begriff des "Rationalismus" bzw. der mit ihm verbundene Prozeß der "Rationalisierung" mit Blick auf die einzelnen gesellschaftlichen und kulturellen Teilbereiche offensichtlich etwas sehr Vieldeutiges beinhalten und insofern nur als theoretische Chiffre für recht unterschiedliche bzw. "eigensinnige" teilsystemspezifische Differenzierungsformen verstanden werden dürfe.[9] Gerade diese Eigengesetzlichkeit bzw. strukturelle Autonomie der einzelnen gesellschaftlichen Wertsphären bzw. Teilsysteme ist es aber, welche nach Maßgabe der neueren soziologischen Analysen dieses funktionalen Differenzierungsprozesses aufgrund der dadurch ermöglichten permanten Leistungssteigerung der einzelnen Teilsysteme zu einem grundsätzlich dynamischen Charakter der Entwicklung moderner Gesellschaften führen muß, der aufgrund der damit verbundenen permanenten Revolutionierung der bestehenden Produktions- und Verkehrsverhältnisse auch innerhalb der neueren historischen Forschung als das Eigentümliche der "neuzeitlich bewegten Geschichte" schlechthin angesehen wird.[10] Solche teilsystemspezifische Rationalisierungsprozesse müssen dabei allerdings, wie bereits von Marx scharfsinnig beobachtet wurde, nicht notwendig zu einer steigenden Rationalität des Gesamtsystems der modernen Gesellschaft führen, sondern können durchaus dessen grundsätzliche "Irrationalität" bzw. Unberechenbarkeit und Unbeherrschbarkeit sogar noch erheblich steigern. Dies zeigen nicht nur die berühmten "schwarzen Freitage" mit ihrer ungeheuren Kapitalvernichtung, sondern auch die zunehmende Selbstgefährdung der modernen Gesellschaft durch spezifische Entwicklungen innerhalb des Teilsystems Wissenschaft oder auch der sukzessive Verbrauch ihrer nicht wieder erneuerbaren ökologischen Ressourcen. Und auch die seit Rousseau, Friedrich Schiller und Hegel auf diesen Typus der funktionalen Differenzierung der modernen Gesellschaft bezogene "alteuropäische" Form der Kulturkritik mit ihrer Beklagung des mit ihr einhergehenden Sinn-, Freiheits- und Gemeinschaftsverlustes stellt ja ihrerseits nur eine nostalgische Form der Selbstbeschreibung der Moderne dar, die trotz des damit verbundenen Rückbezugs auf die griechische Antike oder das christliche Mittelaters so nur auf dem Boden bzw. in dem kategorialen Bezugsrahmen der durch das moderne Zeitalter selbst bewirkten Differenzierungsprozesse möglich geworden ist. Insofern verkörpert auch diese Kulturkritik selbst nur eine weitere - in diesem Fall allerdings ideologiekritische bzw entfremdungstheoretische - Form der Selbstbeschreibung bzw. Selbstunterscheidung der Moderne.[11] Was an dieser Kanonisierung einer spezifisch soziologischen Tradition des Modernitätsverständnisses auffällt, ist zunächst der Umstand, daß zumindest die soziologischen Klassiker selbst so gut wie nie von der Moderne schlechthin im Sinne eines akteurs- bzw. strukturtheoretisch zurechenbaren Gesamtsubjektes oder Systems sprachen, sondern allenfalls von der "modernen Zeit" oder "neuen Zeit" bzw. schlicht "Neuzeit". Oder aber sie sprachen wie im Falle von Ferdinand Tönnies von einer der traditionellen Haus- und Dorfgemeinschaft entfremdeten "Gesellschaft" im Sinne des ökonomischen Systems des Manchesterliberalismus.[12] Max Weber war dagegen an einer universalhistorischen Standortbestimmung und Analyse des "okzidentalen Rationalismus" bzw. des damit verbundenen großen weltgeschichtlichen "Entzauberungsprozesses" interessiert. Bereits dessen intellektuellen Wurzeln in der griechischen Antike und im antiken Judentum weisen darauf hin, daß zumindest Max Weber selbst damit keinen Epochenbegriff zum Ausdruck bringen wollte, sondern nur einen spezifischen Rationalitätsypus, der in den einzelnen gesellschaftlichen Teilbereichen bzw. kulturellen "Wertsphären" unter 
jeweils kontingenten Bedingungen zu völlig unterschiedlichen historischen Zeitpunkten erst allmählich ausgebildet worden ist, dort oft für Jahrhunderte, wenn nicht gar Jahrtausende auf Eis gelegt werden konnte, bis im Laufe der europäischen Neuzeit diese bereichsspezifisch entwickelten Rationalitätspotentiale in einer bis heute gesellschaftstheoretisch noch nicht einwandfrei und überzeugend formulierten Weise wechselseitig aufeinander einzuwirken begannen. Diese spezifische Form der Wechselwirkung zwischen den einzelnen gesellschaftlichen Teilsystemen konnte dabei auf der Ebene der Gesamtgesellschaft gleichsam synergetische Prozesse freisetzen und schließlich einen umfassenden Strukturwandel einleiten, der in den einzelnen Beiträgen des renommierten Wörterbuchs "Geschichtliche Grundbegriffe" unter den übergreifenden Gesichtspunkten der Verzeitlichung, der Politisierung und Ideologisierung der damit verbundenen Modernisierung der historisch-politischen Semantik sowie der Beschleunigung der Veränderungen innerhalb der kollektiven Wahrnehmung der ihr zugrundeliegenden epochalen Erfahrungsgehalte beschrieben wird. Es handelt sich also um genau jenen Transformationsprozeß traditionaler Gesellschaften, den wir heute unter strikt modernisierungstheoretischen Gesichtspunkten insbesondere mit der Alphabetisierung und politischen Demokratisierung, der Kapitalisierung der Wirtschaft und Industrialisierung der Naturaneignung, der Verwissenschaftlichung der modernen Zivilisation und den revolutionären Errungenschaften des technischen Fortschrittes sowie den damit verbundenen demographischen Veränderungen seit Beginn der Neuzeit in Zusammenhang bringen.[13]

Diese terminologische Differenz zwischen der Sprache der soziologischen Tradition einerseits und der zeitgenössischen modernisierungstheoretischen Begrifflichkeit andererseits sowie den hierbei gebrauchten semantischen Unterscheidungen mag ein weiteres Beispiel verdeutlichen: Selbst Georg Simmel, der gemeinhin als zentraler Repräsentant des kulturellen Modernismus angesehen wird, spricht nur an einer einzigen Stelle innerhalb seines umfangreichen Oeuvres von "der Moderne" schlechthin und meinte damit bezeichnenderweise gerade nicht diesen modernisierungstheoretisch beobachtbaren Sachverhalt der Beschleunigung des politischsozialen Wandels im engeren Sinne, sondern eine spezifische Erscheinungsform der ästhetischen Moderne, wie sie um 1900 in der impressionistischen Malerei und Plastik sowie in einer auf eine Steigerung bzw. Verinnerlichung des subjektiven Erlebens abzielenden "Neuromantik" sowie "Dekadenz-" bzw. "Nervenkunst" zum Ausdruck kam. Deren eigentlicher Erfahrungsgehalt zielte dabei allerdings noch nicht auf soziologisch beschreibbare Phänomene im engeren Sinne ab, sondern auf spezifische Erscheinungsformen des modernen Lebens, wie sie zunächst nur einer entsprechenden kunstheoretischen bzw. kulturphilosophischen Reflexion zugänglich waren.[14] Ähnliches gilt im übrigen auch für die von Max Weber beschriebenen Prozesse der "Sublimierung" der spezifisch ästhetischen bzw. erotischen Erfahrungsgehalte der Moderne, deren sozialstrukturelle Korrelate Weber zwar selbst bereits in rudimentärer Form angab, deren "Eigensinn" er aber gleichwohl von einem eindimensionalen Verständnis von "Rationalisierung" zu unterscheiden wußte. Dies ist auch der eigentliche Grund dafür, weshalb er der spezifischen Eigengesetztlichkeit des Ästhetischen und des Erotischen zugleich einen besonderen Namen gab - eben den der "Sublimierung" von im Grunde zutiefts "arationalen" bzw. rein subjektiv zugänglichen Erfahrungsgehalten der menschlichen Existenz -, um diese grundlegenden Unterschiede innerhalb des zunehmenden Autonomwerdens der einzelnen kulturellen Wertsphären zu beschreiben.[15]

Zumindest Max Weber selbst ist also nicht für jene Karikaturen verantwortlich zu machen, die man in der Folgezeit sowohl im Umfeld der Kritischen Theorie als auch der orthodoxen sozialwissenschaftlichen Modernisierungs-forschung aus seinem Werk im Sinne einer ihm nun nachträglich unterstellten Diagnose der Heraufkunft eines eindimensionalen, angeblich primär auf "Zweckrationalität" beruhenden Zeitalters bzw. einer durch solcherart Modernisierung bewirkten Steigerung des gesamtgesellschaftlichen "Fortschrittes" machte. Bei genauerem Hinsehen fällt vielmehr auf, daß die auf Max Webers Werk Bezug nehmende soziologische Modernisierungstheorie ein Produkt der Systemkonfrontationen innerhalb des Zweiten Weltkrieges und des in seinem Schatten entstandenen "Kalten Krieges" ist, in deren Zusammenhang die insbesondere Webers Untersuchung über die "protestantische Ethik" entnommenen normativen Grundlagen der modernen kapitalistischen Arbeits- und Berufsethik sowie die mit ihr verbundenen institutionellen Einrichtungen nun in 
einem legitimatorischen Sinne für die sich zu diesem Zeitpunkt bereits abzeichnende Pax Americana in Anspruch genommen und gegenüber den faschistischen Diktaturen Mitteleuropas und Ostasiens, aber auch gegenüber dem "realen Sozialismus" leninistischer Prägung sowie dem nativistischen und kulturellen "Eigensinn" der verschiedenen Länder der Dritten Welt in einem genuin "ideenpolitischen" Sinne mit zugleich praktischen Konsequenzen geltend gemacht wurden. Die Komplexität, historische Kontingenz und kulturkreishafte Prägung des in Webers Werk beschriebenen Rationalisierungs- und Modernisierungsprozesses wurde dabei aufgrund einer Überbetonung des normativen und universalistischen Gehaltes der okzidentalen Moderne stillschweigend übersehen bzw. gleichsam zu einer quantité négligeable heruntergespielt. Zugleich reduzierte man in diesem Zusammenhang die höchst anspruchsvolle theoretische Form seiner Beschreibung der "Entwicklungsgeschichte" des okzidentalen Rationalismus einseitig auf ein evolutionistisches Modell der Modernisierung von Gesellschaften, dem sich die in den Genuß der westlichen Entwicklungshilfe gekommenen vorkapitalistischen Länder der "Dritten Welt" nun bei Strafe des Entzuges der finanziellen Unterstützung durch die OECD bzw. die Weltbank zu fügen hatten und dessen Scheitern wir seither ebenfalls in vielen dieser Länder als Zeitzeugen beobachten konnten.[16] Nicht zuletzt das Beispiel des Iran sowie vieler arabischer und afrikanischer Länder zeigt insofern bereits seit einiger Zeit in einer sehr eindringlichen Art und Weise, daß es so offensichtlich nicht mehr weiter geht, weshalb sich heute auch die zeitgenössische sozialwissenschaftliche Modernisierungsforschung dazu genötigt sieht, bei Beibehaltung ihrer grundsätzlich universalistisch und evolutionistisch ausgerichteten Prämissen immerhin wenigstens die Möglichkeit eines spezifisch "islamischen", "russischen" und "chinesischen" oder auch "konfuzianischen" Weges in die Moderne als Seitenwege einer dennoch nach wie vor in die gleiche Richtung weisenden Hauptstraße einzugestehen.[17]

Demgegenüber ist die auf die spezifisch westeuropäischen und nordamerikanischen Erscheinungsformen der Moderne Bezug nehmende zeitgenössische soziologische Grundlagendiskussion seit einigen Jahren verstärkt darum bemüht, der insbesondere durch die "poststrukturalistische" bzw. "postmoderne" Provokation der 70er und 80er Jahre bewirkten Verunsicherung des Modernitätsverständnisses der soziologischen Tradition dadurch gerecht zu werden, daß sie nun ihrerseits Differenzierungen im Begriff der Moderne vornimmt, welche dieser vermeintlich "neuen Unübersichtlichkeit" sowohl in einem theoretischen als auch in einem genuin empirischen Sinn Rechnung zu tragen versuchen. Jürgen Habermas hat in diesem Zusammenhang bereits Anfang der 80er Jahre entscheidende Anstöße für diese nun fällig gewordene neuere soziologische Selbstreflexion gegeben, mit denen er den bisher wohl prominentesten Versuch unternahm, die mit dem "Projekt der Moderne" einstmals verbundenen utopischen und normativen Erfahrungsgehalte gegenüber ihrer "postmodernen" Infragestellung zu verteidigen. Habermas konnte dabei gewinnbringend auf die grundbegriffliche Unterscheidung zwischen Sozialstruktur und Kultur bzw. "Modernity" und "Modernism" zurückgreifen, welche der amerikanische Soziologe Daniel Bell bereits 1976 mit seinem Buch "The Cultural Contradictions of Capitalism" in die internationale Diskussion über Wesen und Eigenart der okzidentalen Moderne eingeführt hatte, um den Eigensinn der ästhetisch-expressiven Sphäre gegenüber dem eigentlichen institutionellen Kern der modernen Gesellschaft, wie er vornehmlich im Bereich der Wirtschaft und der Politik verankert ist, zu kennzeichnen. Bells zentrales Anliegen bestand in diesem Zusammenhang darin, einen theoretischen Bezugsrahmen für eine genuin soziologische Analyse der bereits in den 50er Jahren diagnostizierten Heraufkunft eines "postmodernen Zeitalters" sowie der mit ihr verbundenen verschiedenen Erscheinungsformen im Bereich der Kunst, Literatur und modernen Massenkultur angeben zu können. Mit dem Begriff Modernity hatte er dabei jene auf funktionaler Effizienz, demokratischer Gleichheit und Kontrolle beruhenden Prinzipien bezeichnet, die im technischökonomischen und politischen System der modernen Industriegesellschaft westlichen Zuschnittes institutionalisiert sind, denen er nun die spezifisch zeitgenössischen Erscheinungsformen eines ästhetischhedonistischen, auf hemmungslosen Konsum und schrankenlose Selbstverwirklichung ausgerichteten kulturellen Modernismus in einem antinomischen bzw. gar antagonistischen Sinne gegenüberstellte. Und zwar so, daß zugleich deutlich wurde, warum trotz gewisser ursprünglicher charakterologischer "Wahlverwandtschaften" zwischen dem kapitalistischen Unternehmer im Schumpeterschen Sinne und dem modernen Genie-Künstler die durch die ökonomischen Zwänge einer zunehmend sich beschleunigenden Kapitalakkumulation und - 
reproduktion freigesetzte Massenkultur in Gestalt des Massenkonsums sowie der Kulturindustrie notwendig zu einer schleichenden Erosion der normativen Grundlagen des institutionellen Kerns der modernen Gesellschaft führen mußte, der ursprünglich in der bereits von Max Weber beschriebenen Eigenart der puritanischen Arbeitsund Berufsethik seine normative Verankerung besaß. Der "doublebind" bzw. kulturelle Selbstwiderspruch der Moderne besteht Daniel Bell zufolge darin, daß die ihren systemspezifischen Reproduktionsbedingungen zugrundeliegenden Imperative ihrerseits allmählich genau jene Wertvorstellungen in Frage stellen mußten, welche das "Projekt der Moderne" historisch überhaupt erst ermöglicht und für eine gewisse Zeit lang auch in einem institutionellen Sinne bestandsfähig gemacht hatten. Seit diesem inzwischen auch von zeitgenössischen soziologischen Theoretikern wahrgenommenen Auseinanderdriften des "Modernismus" und der "Modernität" fallen aber die sozialstrukturellen und die kulturellen Beschreibungen der Moderne bzw. die soziale Stellung des einzelnen und sein persönlicher "Kulturstil" zunehmend auseinander, wobei die einstmals als linearer Fortschritt gedachte zukünftige Entwicklung der Moderne zumindest im Bereich der Kultur jetzt die mythologische Struktur einer zyklischen Veränderung bzw. "ewigen Wiederkehr des Gleichen" annimmt, die in den an dem Wechsel der Mode orientierten modernen Erscheinungsformen des Massenkonsums, der Kulturindustrie sowie der ästhetischen Stilisierung des Alltagslebens ihren Niederschlag findet.[18]

Diese neuerliche, nun spezifisch kulturindustriell inszenierte Aufwertung des Vergänglichen und Kontingenten hatte in der Folgezeit eine zunehmende Neigung zum kulturellen Synkretismus begünstigt, der in den beiden vergangenen Jahrzehnten im Neohistorismus eines nun als "postmodern" empfundenen Denkens geradezu affirmiert und schließlich sogar zum "epochalen" Kennzeichen einer neuen Form der kulturellen Vergesellschaftung stilisiert worden ist. Seither sprechen nicht nur die Kunst und die Industrie unterschiedliche Sprachen, sondern eben auch der Kunst-, Literatur- und Medienwissenschaftler einerseits sowie der nach wie vor an den Grundprämissen der "klassischen Moderne" orientierte Soziologe und Sozialwissenschaftler andererseits. Und wie bereits Walter Benjamin und Theodor W. Adorno feststellen konnten, verliert die Kunst ihrerseits im Zuge ihrer massenmedialen Vermarktung zunehmend jene "Aura" bzw. "erlösende Funktion", die ihr einstmals gegenüber den Zwängen des alltäglichen Lebens noch in einem kritischen und insofern eben gerade nicht "affirmativen" Sinn zugesprochen worden ist.[19] Andererseits weisen inzwischen nicht nur

Modernitätstheoretiker wie Daniel Bell, sondern auch entsprechende neuere Untersuchungen von soziologischen Autoren wie Pierre Bourdieu, Michel Maffesoli, Mike Featherstone und Scott Lash oder in der Bundesrepublik Deutschland z.B. Gerhard Schulze darauf hin, daß die einstmals "auratische" Sphäre der Kunst in Gestalt der Kulturindustrie und einer durch sie geprägten ästhetisch-hedonistischen Stilisierung der alläglichen Lebensformen nun selbst zu einem wesentlichen "Unterbau" der modernen Gesellschaft geworden ist bzw. eine ihrer zentralen Reproduktionsbedingungen darstellt, die in einer zunehmenden "Verbürgerlichung" bzw. Institutionalisierung der einstmals in einem noch "systemtranszendierenden" Sinne gedachten Normen und Werte der internationalen Jugendbewegung der 60er Jahre zum Ausdruck kommt.[20] Aus genau diesem Grund zogen deshalb auch einige sich eher dem politischen und kulturellen Konservatismus verpflichtet fühlende soziologische Beobachter dieses Transzendenzverlustes der Kunst bzw. der durch sie geprägten bohemienhaften Lebensformen wie Peter L. Berger, Robert N. Bellah und Daniel Bell bereits recht früh, d.h. unmittelbar in Reaktion auf den durch die Jugend- und Studentenbewegung der sechziger Jahre in den westlichen Industrienationen bewirkten "Wertewandel" den Schluß, daß mit diesem Verlust der Transzendenz konsequenterweise auch eine Renaissance von genuin neuen religiösen Bewegungen und entsprechend relgiös gefärbten Formen der "Sinnsuche" bzw. des "subjekiven Erlebens" zu erwarten sei, die seitdem übrigens nicht nur in den USA, sondern inzwischen auch in Europa festgestellt werden kann.[21]

Jürgen Habermas hat dieses Ausanderfallen von Sozialstruktur und Kultur bzw. der auf sie bezogenen Beschreibungen der Moderne vor dem Hintergrund der normativen Grundlagen seiner eigenen "Theorie des kommunikativen Handelns" dergestalt gedeutet, daß das utopisch-emanzipatorische Potential des im 18. Jahrhundert entstandenen und dem Geist der europäischen Aufklärung verpflichteten "Projekts der Moderne" mit dem real zu beobachtenden Modernisierungsprozeß offensichtlich nicht mehr unmittelbar in Deckung zu bringen 
ist. Vielmehr haben sich mit der zunehmenden Entfaltung des "Eigensinns" der einzelnen gesellschaftlichen Teilsysteme wie dem der Wirtschaft, der Politik, Wissenschaft und der Kunst die Diskrepanzen zwischen einer jeweils teilsystemspezifisch verselbständigten Expertenkultur einerseits und der im Alltagshandeln verankerten kommunikativen Struktur der Lebenswelt andererseits offensichtlich dermaßen verstärkt, daß die emanzipatorischen Gehalte der in den einzelnen Basisinstitutionen der Gesellschaft sedimentierten bürgerlichen Normen und Wertvorstellungen heute nicht mehr unmittelbar, d.h. in einem "entsublimierten", sondern eben nur noch in einem "sublimierten" bzw. sublimen Sinn für die Verständigungs- und Orientierungsprobleme im Bereich des alltäglichen Handelns fruchtbar gemacht werden können. Der surrealistische Versuch, das noch im auratischen Kunstwerk zum Ausdruck kommende Versprechen auf eine prinzipielle "Erlösung" der Menschheit von den Zwängen der Rationalisierung bzw. einer "Versöhnung" der in sich gespaltenen Moderne nun in Gestalt einer radikalen Aufhebung des spezifisch modernen Gegensatzes zwischen Kunst und Leben gleichsam terroristisch in die Alltagspraxis einzuführen, wie dies ja bekanntlich André Breton empfahl, haben vielmehr deutlich gemacht, daß auch die Kunst nur um den Preis ihrer eigenen Selbstaufgabe sowie des Verlustes ihres kritisch-emanzipatorischen Gehaltes in einer ästhetischen Formung des Alltagshandelns bzw. einer massenkulturellen Stilisierung von vormals bohemienhaften Lebensweisen aufgehoben werden kann.[22] Mit anderen Worten: Nicht nur die Wissenschaft, sondern gerade auch die Kunst verfehlt das ihr einstmals zugeschriebene Versprechen auf Aufklärung und Beförderung des gesellschaftlichen Fortschrittes, wenn sie sich nicht selbst an dem fortgeschrittensten Stand des gesamtgesellschaftlichen Rationalisierungs- und Differenzierungsprozesses orientiert, sondern diesen aktionistisch und publikumswirksam zu unterlaufen versucht. Adornos Diktum, daß moderne Kunst nur dann nicht den Anschluß an den gesamtgesellschaftlichen Fortschritt verliert - und sei es auch ein technologisch und kapitalistisch induzierter -, wenn sie sich selbst als "Kunst fortgeschrittensten Bewußtseins" begreift, die sich in ihrer eigenen souveränen Materialbeherrschung zugleich der ökonomischen Logik des "Hochindustrialismus" gewachsen zeigt,[23] wird deshalb auch für Habermas zur Garantie dafür, daß nur in einer reflektierten Form der Vermittlung von Expertenwissen und Massenkultur der aufklärerische Gehalt des Projektes der Moderne bewahrt und auf der Grundlage einer genuin kommunikativen Ethik der Verständigung über die "Signatur der Zeit" zugleich für eine "emanzipatorische" Orientierung der Lebenspraxis fruchtbar gemacht werden kann. Modernisierung der Moderne bedeutet für Habermas insofern nicht "postmoderner" Abschied von dem mit der europäischen Aufklärung verbundenen Glücksversprechen, sondern die Einführung einer grundlegenden Unterscheidung im Begriff des Rationalen selbst, welche das Rationalitätspotential von praktischen Verständigungsprozessen gegenüber jenen systemspezifischen Rationalisierungsprozessen abgrenzt, wie sie zum Beispiel im Bereich der Wirtschaft, Verwaltung und Wissenschaft im Sinne von rein technischen Effizienzsteigerungen institionell verankert sind, ohne daß dies notwendig zu einer grundsätzlichen Unvereinbarkeit der mit beiden Rationalitätsformen verbundenen Sinngehalte führen muß.[24]

Die von Habermas konstatierte Form einer grundlegenden, für sie als solche überhaupt erst historisch konstitutiven Selbstunterscheidung der Moderne zehrt noch von dem Glauben bzw. dem Optimismus, daß sich technologische und kommunikative Formen der Rationalität auf der Ebene von übergreifenden formalen Prozeduren einer normativ idealisierten Verständigungspraxis miteinander vermitteln lassen und ihr aufklärerischer Gehalt zugleich für eine nichtregressive Form der Gestaltung des ästhetisch-expressiven Verhaltens innerhalb der modernen Massenkultur in Anspruch genommen werden kann. Das "Projekt der Moderne" ist ihm zufolge deshalb nur dann in einer selbstreflexiven Form zu retten und für die weiteren noch anstehenden Verständigungsprozesse in den fortgeschrittenen Industriegesellschaften fruchtbar zu machen, wenn sein utopisch-normativer Gehalt nicht zugunsten einer "alt"-, "jung"- oder gar "neukonservativen" Verabschiedung von eben diesem Glücksversprechen aufgegeben wird, sondern gerade kontrafaktisch, d.h. mit den Mitteln einer sich des Mediums der Öffentlichkeit bedienenden und die Standards der modernen Wissenschaft und Kunst bewahrenden Aufklärung über die mit den normativen Grundlagen des modernen Zeitalters verbundenen, gesamtgesellschaftlich bisher allerdings noch uneingelösten Rationalisierungspotentiale geltend gemacht werden kann. Insofern steht und fällt die Habermassche Form der Zeitdiagnose bzw. 
Unterscheidung der Moderne mit der kontrafaktischen Wirkung jenes "zwanglosen Zwangs des besseren Arguments", den Habermas seit über einem Vierteljahrhundert gegenüber den Gebildeten unter seinen Verächtern zu verteidigen versucht.

Zwei Gesichtspunkte möchte ich im folgenden aus Habermas' Theorie des kommunikativen Handelns herausgreifen, die uns für eine Klärung der von ihm angesprochenen Formen der Selbstunterscheidung der Moderne weiterführen. Der eine betrifft dasjenige von ihm beschworene Rationalisierungspotential, welches genau dann zur Entfaltung kommt, wenn die Moderne nicht für historisch überholt erklärt wird, sondern Modernisierungsprozesse dergestalt aufeinander bezogen gedacht werden, daß wir allererst von einer in sich reflektierten bzw. reflexiven Moderne sprechen können. Eine solche Form der Selbstbeschreibung bzw. Selbstunterscheidung der Moderne bezieht sich also bereits ihrem eigenen grundbegrifflichen Verständnis nach nur noch auf sinnhafte Operationen und Differenzierungen, die keine wie auch immer gearteten, ihr extern vorgegebenen Restbestände an überlieferten Traditionen mehr in Anspruch nehmen. Das heißt, sie stellt dergestalt dann wirklich eine genuin "autopoietische" bzw. selbstgenügsame Form der Systembildung dar, welche sich nurmehr vermittels eigener systemspezifischer Prozesse auf ausschließlich von ihr selbst geschaffenen Grundlagen reproduziert.[25] Der zweite Punkt bezieht sich dagegen auf die bereits von Autoren wie Adorno und Habermas aufgestellte und unlängst auch von Niklas Luhmann bekräftigte Forderung, daß auch die moderne Kunst nur dann einen wesentlichen Beitrag zur Selbstbeschreibung und Selbstunterscheidung des mit dem Signum "Moderne" gekennzeichneten Zeitalters zu leisten vermag, wenn sie in der historischen Entfaltung ihrer spezifisch eigenen Entwicklungslogik bzw. Eigengesetzlichkeit zugleich jener Selbstreflexivität des gesamtgesellschaftlichen Modernisierungsprozesses in einem konstitutiven Sinne Rechnung zu tragen vermag, wie sie bereits von Marx am Beispiel der Entfaltung des modernen kapitalistischen Systems der Produktion und Reproduktion exemplarisch beschrieben worden ist und heute im Rahmen einer autopoietisch gewendeten Form der sozialwissenschaftlichen Systemanalyse als Kennzeichen der Konstitution und Beschreibung von Sinnsystemen schlechthin angesehen wird.[26] Der erste Gesichtspunkt betrifft dabei jene soziologische Grundlagendiskussion bezüglich der Selbstunterscheidung der Moderne in eine einfache und eine reflexive Moderne, wie sie unter anderem in den neueren Arbeiten von Ulrich Beck, Anthony Giddens sowie Scott Lash zum Ausdruck kommt. Der zweite Punkt betrifft dagegen jene ästhetischen Dimensionen einer solchen reflexiven Form der Modernisierung, welche die innersoziologische Fachdiskussion bezüglich der epochalen Eigenart der Moderne zugleich in ein fruchtbares Verhältnis zu den entsprechenden Grundlagenreflexionen in den benachbarten geistes- und kulturwissenschaftlichen Disziplinen zu bringen vermag. Beide Strategien im Umgang mit den im Begriff der Moderne angesprochenen epochalen Erfahrungsgehalten sollen zugleich als Antwort auf die sich in diesem Zusammenhang aufdrängende Frage verstanden werden, welchen möglichen Beitrag die zeitgenössische soziologische Forschung und Theoriebildung denn nun ihrerseits zur Klärung der mit der inzwischen auch nicht mehr ganz so neuen Proklamation eines vermeintlichen "Traditionsbruches" innerhalb der Moderne in Gestalt der Heraufkunft eines ihrem eigenen Schoß entsprungenen und sie angeblich radikal in Frage stellenden "postmodernen" Zeitalters zu leisten vermag.

Im deutschen Sprachraum ist es neben Jürgen Habermas insbesondere Ulrich Beck zu verdanken, daß das orthodoxe Modernitätsverständnis der soziologischen Tradition durch eine wesentlich differenziertere Betrachtungsweise des mit dem Begriff der Moderne verbundenen gesamtgesellschaftlichen Rationalisierungspotentials ergänzt und zum Teil auch erheblich modifiziert worden ist. Beck macht in seinen eigenen Arbeiten nämlich darauf aufmerksam, daß jener mit der im Laufe des 19. Jahrhunderts entstandenen Industriegesellschaft gleichgesetzte Begriff der Moderne seinerseits nur Ausdruck einer "halbierten Moderne" sei. Denn diese beruhe auf Voraussetzungen, die spezifische ständische Eigenschaften der modernen industriellen Produktionsweise wie z.B. die nationalstaatliche Organisation der Volkswirtschaft, den weitgehenden Ausschluß der Frauen und Kinder aus dem Erwerbsleben, die Existenz von intakten Kleinfamilien als Reproduktionsbedingung der männlichen Ware Arbeitskraft, eine auf Klassenbildung beruhende Form der sozialen Ungleichkeit sowie eine systemkonstitutive Differenzierung zwischen der Welt der Experten und jener der Laien zur unabdingbaren Voraussetzungen haben. Die im wesentlichen auf vermeintliche "Naturkategorien" 
wie der geschlechtlichen Differenzierung und dem Generationsunterschied beruhende Einteilung der Bevölkerung in Lohnarbeiter und Nichterwerbstätige widerspreche aber dem grundlegend universalistischen Charakter der modernen Warenökonomie, deren Prinzipien nur dann voll greifen, wenn diese ständischen Unterscheidungen ihrerseits zugunsten einer durch den sukzessiven Einbezug der gesamten Bevölkerung in den Arbeitsmarkt bewirkten zunehmenden Individualisierung der Berufskarrieren und ihrer sozialisatorischen Voraussetzungen sowie der intimen Lebensformen historisch außer Kraft gesetzt werden.[27]

Genau diesen Fall sieht Beck nun im heutigen Stadium eines reflexiv gewordenen Modernisierungsprozesses gegeben, dessen weitere Entwicklung seither nur noch an der Bewältigung der von der industriellen Moderne bewirkten Nebenfolgen orientiert ist und insofern überhaupt erst das im Begriff der Moderne selbst bereits implizit enthaltene Autonomiepostulat sowie gesellschaftliche Rationalisierungspotential zur vollen Entfaltung gebracht hat. Enttraditionalisierung der Schichtungsformen der Sozialstruktur und der Beziehungen zwischen den Erwachsenen und den Kindern sowie zwischen den Geschlechtern, Repolitisierung der Privatsphäre im Sinne einer ausschließlich an den Marktchancen orientierten Strukturierung des individuellen Lebenslaufes, Globalisierung der zivilisatorischen Risiken der durch die moderne Wissenschaft und Technik bewirkten Veränderungen der natürlichen Grundlagen aller menschlichen Existenz sowie eine dadurch bewirkte basisdemokratische und gegenkulturelle Infragestellung der traditionellen Expertokratie in Wirtschaft, Politik und Wissenschaft durch eine von zahlreichen "Gegenexperten" bzw. den Laien selbst getragene "Palaver-Kultur" treten so schließlich zunehmend an die Stelle jener "ständischen" Prinzipien, welche die "klassische Industriegesellschaft" kennzeichneten und nun den Weg hin zu einer anderen, jetzt allerdings überhaupt erst ihrem eigenen Begriff gerecht werdenden und mithin reflexiv gewordenen Moderne freimachen. Beck bringt diese neue Form der Selbstunterscheidung der Moderne dabei durch den Gegensatz zwischen einer einfachen, d.h. auf die der Industriegesellschaft noch ständisch vorgegebenen Traditionen und einer auf die grundlegenden Prämissen der Industriegesellschaft selbst bezogenen reflexiven Form der Modernisierung zum Ausdruck, welche nun in einem gleichsam systemtranszendierenden Sinne "die Prinzipien der Moderne gegen ihre industriegesellschaftliche Halbierung einklagt".[28]

An Becks Konstruktion besticht die relative theoretische Anspruchslosigkeit, mit der er eine neue Form der Selbstunterscheidung der Moderne in die öffentliche Diskussion eingebracht hat. Nicht zufällig ist er in den letzten Jahren sowohl im Fach selbst als auch innerhalb des gehobenen Feuilletons zum Liebling einer breiteren Öffentlichkeit geworden, der es aus berechtigten Gründen ohnedies immer schwerer fällt, den beträchtlichen Abstraktionsgrad sowie das hierbei inzwischen erreichte Anspruchsniveau der internationalen soziologischen Theoriediskussion bezüglich Wesen und Eigenart der Moderne nachzuvollziehen. Dies spricht weder gegen Ulrich Beck selbst noch gegen das hier zur Diskussion stehende Fach, sondern nur gegen die von Autoren wie Beck und neuerdings auch Dirk Käsler prominent vertretene und bewußt genährte Hoffnung, daß sich das gerade auch bezüglich des Verhältnisses der soziologischen Profession zu ihrer Umwelt erreichte strukturelle Gefälle zwischen Expertenkultur und Laientum zugunsten eines "Letztlich sind wir ja alle Betroffene" voluntaristisch aus der Welt schaffen oder mit den Mitteln der Massenmedien publikumswirksam in Form von

Selbstverbrennungsakten herunterspielen lasse.[29] Dies gelingt nämlich, wie Jürgen Habermas deutlich gemacht hat, ohnedies nur um den Preis einer kulturellen Regression bzw. "repressiven Entsublimierung" der spezifischen Erfahrungsgehalte der Moderne. Vor Aktionismus sei also an dieser Stelle ausdrücklich gewarnt, und sei es auch nur einer, welcher dieses Mal in dem scheinbar harmlosen Gewand von zunehmend wie Pilze aus dem Boden schießenden selbsternannten "Experten" oder basisdemokratisch erzwungenen "Ethikkommissionen" auftritt. Auch dies mag aber als eine neue Erscheinungsform der Selbstunterscheidung der Moderne verstanden werden: dieses Mal allerdings in Gestalt der Globalisierung von "Betroffenheit" sowie der Universalisierung von "Entscheidungskompetenz"!

Der britische Soziologe Anthony Giddens teilt mit Beck zwar viele Prämissen und empirische Einzelbeobachtungen dieser nun offensichtlich endgültig selbstreflexiv gewordenen Modernisierungstheorie, meint im Unterschied zu Beck dabei aber nicht ein "reflexartiges" Reagieren des Gesellschaftssystems auf seine 
eigenen Nebenfolgen vor dem Hintergrund eines systembedingten Nicht-Wissens über die möglichen Risiken der durch die industrielle Moderne erzeugten Gefährdungen der Spezies Mensch, sondern ein Reflexivwerden der Moderne mit Hilfe eines genuinen Expertenwissens über die Grundlagen, Folgen und strukturellen Probleme sowie Antinomien und Paradoxien solcher Modernisierungsprozesse. Allein eine solche institutionalisierte Form der Reflexivität ist Giddens zufolge nämlich noch in der Lage, jene durch den zunehmenden Globalisierungsprozeß bewirkte space-time-distanciation bzw. raumzeitlichen "Abstandsvergrößerung" zwischen dem alltäglich Erlebbaren und dem systemisch induzierten sozialen und kulturellen Wandel zu überbrücken. Und zwar dergestalt, daß eine nun strukturell mit dem Dissens rechnende, gleichwohl ein spezifisches institutionelles Vertrauen genießende Expertenrationalität dem alltäglichen Erleben und Handeln wieder diejenigen Orientierungen vermittelt, die sich nicht mehr bruchlos über den Konsum von Massenmedien - und sei es auch die F.A.Z. - oder die lebensweltliche Organisierung von Betroffenheitskulten gewinnen lassen. Gleichwohl sieht auch Giddens, daß eine solche institutionalisierte Form von sozialer Reflexivität erst dann das Vertrauen breiterer Gesellschaftsschichten zu gewinnen vermag, wenn sie sich selbst dem durch alternative Formen des Expertentums ausgeübten Zwang zur öffentlichen Diskussion und Begründung ihrer eigenen Situationsdeutungen stellt. Ein solcherart gewonnenes "aktives Vertrauen" wird ihm zufolge zur Gewähr dafür, daß über die prinzipielle Anerkennung der Vielheit möglicher Beobachterstandpunkte in der Moderne so etwas wie ein "reflexive citizen" als konkrete Utopie und Träger einer wirklich durchgreifenden gesellschaftlichen Reformpolitik auf dem Boden einer durch die prinzipielle Möglichkeit des Dissenses gestärkten Expertenkultur entstehen bzw. bewußt erzogen werden kann. Und eine solcherart wahre post-traditionale Gesellschaft würde schließlich ihre eigenen Traditionsbildungen weder in der Vergangenheit suchen noch in einem mimetischen Wiederholungszwang zum Ausdruck bringen, sondern im Modus eines offenen Dialoges bzw. BargainingProzesses selbst erzeugen, von dem im übrigen seit einiger Zeit nicht zuletzt ja auch die Tradition des Geschlechterverhältnisses und die ihm bisher historisch zugrundeliegenden Unterscheidungen bzw.

"Leitdifferenzen" betroffen sind.[30]

Beck und Giddens sind neben Habermas zwei prominente zeitgenössische soziologische Autoren, die sich mit den Mitteln ihres eigenen Faches bewußt den durch die Proklamation eines "postmodernen Zeitalters" bewirkten Provokationen und Verunsicherungen des Zeitgeistes gestellt und diese für ihre eigenen wissenschaftlichen Arbeiten fruchtbar zu machen versucht haben. Ihre eigenen Formen der theoretischen Anaylse der Unterscheidungen der Moderne leiden jedoch im Unterschied zu der von Habermas unternommenen Ehrenrettung des "Projektes der Aufklärung" daran, daß sie den im Postmodernismus zum Ausdruck kommenden spezifischen ästhetischen Erfahrungsgehalt der Moderne nicht wirklich aufgenommen und für eine Bestimmung der ästhetischen Dimensionen solcherart reflexiv gewordener Modernisierung zugrunde gelegt haben. Insofern stellen ihre Arbeiten auch kein ernstzunehmendes Gesprächsangebot für eine wirklich interdisziplinäre Diskussion der in den ästhetischen und literarischen Beschreibungsformen des kulturellen Modernismus bzw. im Postmodernismus zum Ausdruck kommenden Unterscheidungen dar. Die Welt des Sozialen und die der Kultur stehen bei ihnen letztendlich immer noch unversöhnt gegenüber, ohne daß deutlich wird, wie die durch einen solchen Bruch zwischen der sozialstrukturellen und der kulturellen Beschreibungen der Moderne verursachte babylonische Sprachverwirrung durch eine spezifisch soziologische Analyse der mit den ästhetischen Erfahrungsgehalten der Moderne bzw. Postmoderne verbundenen Irritationen wenn schon nicht überwunden, so doch zumindest in einem genuin zeitdiagnostischen Sinne fruchtbar gemacht werden kann. Insofern ist der von dem britischen Soziologen Scott Lash getroffenen Feststellung zuzustimmen, daß bei dieser Art der "Übertragung der kulturtheoretischen Begrifflichkeit in den Kontext soziologischer Theorie ... die kulturellen und speziell die ästhetischen Aspekte der Moderne und des Problems der Reflexivität weitgehend unter den Tisch gefallen (sind)".[31] Wenn also die von vielen Beobachtern der internationalen Kulturszene der letzten zwanzig Jahre wiederholt geäußerte Feststellung zutreffen sollte, daß die Entstehung des Postmodernismus sowie sein nicht zu übersehender Erfolg innerhalb der modernen Geisteswissenschaften und der zeitgenössischen Massenkultur ohnedies nur auf dem Boden eines tieferen Verständnisses der epochalen Eigenart der ästhetischen Moderne verstanden werden kann, so stellt sich die Frage, warum der Sphäre des Ästhetischen heute auch im Rahmen einer 
strikt soziologischen Betrachtungsweise überhaupt eine solche Bedeutung bei einer Klärung der Grundprinzipien dieser reflexiv gewordenen Moderne zugesprochen werden muß. Ich möchte diese genuin ästhetischen Implikationen reflexiver Modernisierung deshalb abschließend in Form eines kurzen historischen Rückblickes auf den eigentlichen Anteil des Ästhetischen bei der Selbstunterscheidung bzw. den damit verbundenen Transformationen der Moderne sowie in Gestalt eines Verweises auf die Stellung des Kunstsystems innerhalb einer nun wirklich zeitgemäßen, d.h. im wahrsten Sinne des Wortes auf der Höhe der Zeit stehenden soziologischen Beschreibung der Moderne deutlich machen.

Ästhetische Bedeutungsgehalte spielten bereits zu Beginn der Neuzeit eine entscheidende Rolle bei der historischen Formierung eines spezifisch epochalen Modernitätsbewußtseins, in dem die gegenwärtige Epoche als ein definitiver Bruch mit der Tradition bzw. der Vergangenheit zum Ausdruck gebracht wurde. In wortgeschichtlicher Hinsicht taucht der begriffliche Gegensatz antiqui/moderni bereits gegen Ende des 5. Jahrhunderts n. Chr. auf, wobei der lateinische Ausdruck modernus sowohl in der Bedeutung des "Neuen" als auch des "Gegenwärtigen" gebraucht wurde. In beiden Verwendungsweisen sollte die Überwindung der griechischen und römischen Antike durch das beginnende christliche Zeitalter kenntlich gemacht werden.[32] Als Epochenbezeichnung taucht der Begriff modernitas im Hochmittelalter bereits 1075 auf, um nun im Rahmen einer kirchlichen Reformbewegung eine Zwischenzeit bzw. media aetas auf dem Weg hin zu einer höheren Stufe bzw. reformatio des religiösen Lebens zu kennzeichnen. Bei den moderni des 12. Jahrhunderts wird das Verhältnis zur antiquitas dagegen dergestalt bestimmt, daß die christliche Gegenwart sich jetzt selbst als fruchtbarer Boden, aber zugleich auch als konsequente Weiter- und Höherentwicklung des bereits von den alten Meistern geschaffenen intellektuellen und kulturellen Erbes der Menschheit verstand.[33] Im Sinne eines sich ausschließlich nur noch auf sich selbst beziehenden Zeitalters wird die Gegenwart dagegen erst gegen Ende des 17. Jahrhunderts innerhalb der französischen Frühklassik beschrieben. Und zwar in der berühmten Querelle des Anciens et des Modernes, in der die Freunde der alten Meister und die Verfechter einer genuin neuen Kultur unerbittlich aufeinanderstießen. Bezeichnenderweise ist dieser Streit zwischen den "Alten" und den "Neuen" im Rahmen einer ästhetischen Diskussion geführt worden: nämlich bezogen auf die normative ästhetische Frage, ob sich die Kunst des Altertums überhaupt noch überbieten lasse, d.h. ob eher den Werken der alten oder aber der neuen Meister eine vorbildliche Funktion für die zeitgenössische Kunst und Literatur zugesprochen werden muß. Man einigte sich in diesem Streit dann schließlich darauf, daß nicht nur die gegenwärtigen Künstler und Schriftsteller Werke von klassischem Rang hervorzubringen vermögen, sondern daß auch den Werken der Alten nach wie vor eine bleibende ästhetische Bedeutung zugesprochen werden muß. [34]

Mit dieser historischen Relativierung des ästhetischen Ideals der Schönheit war aber nicht nur die Beliebigkeit bzw. die polemische Umkehrbarkeit des Verhältnisses zwischen den "Alten" und den "Neuen" deutlich geworden, da die "Jungen" sich jetzt ja ebenfalls bereits als "Alte" von normativer Dignität empfanden, sondern auch die mögliche Geburt einer neuen Klassik, welche nicht mehr des normativen Bezuges auf vorgegebene Traditionen bedarf, sondern die ihr zugrundeliegenden ästhetischen Ideale in Form eines selbstgenügsam gewordenen kunsttheoretischen und geschichtsphilosophischen Diskurses gleichsam selbst erst "autopoietisch" erzeugt. [35] Zugleich ist spätestens seit dieser ästhetisch-geschichtsphilosophischen Grundlagendiskussion zu Beginn der Französischen Klassik deutlich geworden, daß mit dieser Gegenüberstellung einer dem Ideal der neuzeitlichen Wissenschaft sich verpflichtet fühlenden Vorstellung eines möglichen historischen Fortschrittes gegenüber den Werken der alten Meister einerseits und der nach wie vor an der zeitlosen und mithin ewigen Bedeutung der klassischen Kunst orientierten ästhetischen Betrachtungsweise andererseits eine folgenschwere "Aufsprengung des alteuropäischen Rationalitätskontinuums" in dem Sinne verbunden gewesen ist, daß jene der Sphäre der Wissenschaft und der Kunst zugrundeliegenden unterschiedlichen Wertvorstellungen als unvereinbar empfunden wurden und sich deshalb auch zunehmend "eigensinniger" auseinanderzuentwickeln begannen.[36] Diese in der Auseinandersetzung mit der antiken Kultur gewonnene ästhetische und poetologische Gegenwartsbeschreibung ging dabei zeitgleich einher mit einem neuen, nun genuin geschichtsphilosophischen Verständnis von Modernität, das allerdings zur Kennzeichnung der hierbei zum Ausdruck kommenden epochalen 
Erfahrungsgehalte die semantisch bereits ausdifferenzierte und mithin zur Verfügung stehende Modernitätsterminologie selbst so gut wie gar nicht gebrauchte. An ihre Stelle traten vielmehr politische Kampfbegriffe wie z.B. Aufklärung, Fortschritt und Neuzeit, die nun in polemischer Weise einem jetzt als "dunkel" und "rückständig" empfundenen "Mittelalter" gegenüberstellt wurden. Als Kollektivsingular zur Kennzeichnung eines Epochenbegriffs taucht dagegen "Die Moderne" erst seit der Mitte des 19. Jahrhunderts in den einzelnen europäischen Nationalsprachen auf, und zwar ebenfalls im Rahmen einer ästhetischen und geschichtsphilosophischen Selbstreflexion der zeitgenössischen künstlerischen Produktion vor dem Hintergrund der generationsspezifischen Erfahrung eines Scheiterns der mit den bürgerlichen Idealen des Aufklärungszeitalters verbundenen Hoffnungen auf eine revolutionäre Umgestaltung der bestehenden politischen und sozialen Verhältnisse.[37] Es ist aber gerade dieser neue, nun genuin ästhetische Begriff der Moderne, dessen spezifische temporale Erfahrungsgehalte sich nicht mehr ohne weiteres mit einem am Modell des "Fortschrittes" orientierten Geschichtsverständnis zur Deckung bringen lassen, sondern fortan eine Zweideutigkeit bzw. Ambivalenz im Modernitätsverständis zum Ausdruck bringen, die bis heute nachwirkt. Denn während sich der technisch-industrielle Entwicklungsprozeß und manchmal auch die Veränderung der politisch-sozialen Strukturen nach wie vor in Fortschrittskategorien beschreiben lassen, bezieht sich dieses ästhetisch artikulierte Modernitätsverständnis seither offensichtlich auf ganz andere Unterscheidungen als die zwischen "Tradition" und "Fortschritt" bzw. "Stillstand" und "Entwicklung". Das moderne ästhetische Selbstbewußtsein zeichnet sich demgegenüber vielmehr durch eine Reihe von sich schnell ablösenden paradoxen Verhältnisbestimmungen zwischen dem "Alten" und dem "Neuen" aus, die sich nun nicht nur gegen jegliches genuin historische Verständnis der mit dieser semantischen "Leitdifferenz" zum Ausdruck gebrachten Erfahrungsgehalte richten, sondern auch gegen den Versuch, das dergestalt autonom gewordene moderne Kunstsystem wieder durch externe geschichtsphilosophische Bindungen bzw. politische Parteinahmen auf ein wie auch immer geartetes Telos der gesamtgesellschaftlichen Entwicklung zu verpflichten. Die moderne Kunst und die mit ihr verbundenen Beschreibungen der Moderne entfalten ihre teilsystemspezifische Autonomie vielmehr fortan in einem permanenten Spiel der Umkehrung zwischen dem "Alten" und dem "Neuen" sowie in Gestalt einer beständigen Veränderung bzw. Auslotung der prinzipiell möglichen Grenzziehungen zwischen dem, was eigentlich überhaupt noch als Kunst anzusehen ist bzw. was nicht.[38]

In dieser Form der Selbstbeschreibung reflektiert das moderne Kunstsystem aber zugleich eine Reihe von grundlegenden Unterscheidungen, die auch in verschiedenen anderen Modernitätsdiskursen zum Ausdruck kommen und die insofern wenigstens eine spezifisch formale Eigenart der Moderne kennzeichnen. Indem nämlich insbesondere die zeitgenössische Kunst den Unterschied zwischen Kunst und Nicht-Kunst bzw. Kunst und Alltag fast bis zur Unkenntlichkeit aufhebt bzw. dessen Un-Möglichkeiten in einem konsequenten Sinne durchbuchstabiert, der sich ohnedies nur noch Eingeweihten bzw. einer immer abstrakter und intellektuell anspruchsvoller werdenen Kunsttheorie erschließt, reflektiert sie zugleich ein zentrales Strukturprinzip, welches der Moderne auch von der neueren soziologischen Forschung allgemein zugesprochen wird: nämlich das der funktionalen Differenzierung sowie die mit diesem Strukturprinzip verbundenen Paradoxien und Pathologien der modernen Gesellschaft. Und indem die zeitgenössische Kunst das immer paradoxer werdene Verhältnis zwischen dem "Alten" und dem "Neuen" fast schon bis zur Ununterscheidbarkeit durchbuchstabiert, macht sie ihrerseits in einer ihre eigene gesellschaftliche Autonomie bestätigenden Weise deutlich, welche spezifischen temporalen Erfahrungsgehalte mit dem Begriff der Moderne in einem nun nicht mehr historischen bzw. geschichtsphilosophischen, sondern genuin paradoxen bzw. ausschließlich gegenwartsbezogenen Sinne verbunden sind.

Die in dieser Form der Selbstunterscheidung der Moderne zum Ausdruck kommenden zeitlichen Paradoxien lassen sich am Beispiel des Verhältnisses zwischen der Moderne und der sogenannten Postmoderne relativ einfach verdeutlichen. Während nämlich noch Charles Baudelaire, dem wir wohl den ersten voll ausformulierten und durchreflektierten Begriff der ästhetischen Moderne verdanken, die Eigenart der zeitgenössischen Kunst gerade darin identifizierte, daß sie auch noch in der flüchtigsten und oberflächlichsten Erscheinungsform des modernen Lebens einen bleibenden bzw. "ewigen" Bedeutungsgehalt zu entziffern vermag und dabei zugleich 
ihre eigene zukünftige "Klassik" antizipiert, wird im sogenannten "Diskurs der Postmoderne" das Verhältnis zwischen Vergangenheit, Gegenwart und Zukunft, welches ein Kunstwerk im Rahmen seiner individuellen Karriere als Entwicklungsstadien durchläuft, gerade umgekehrt: Denn innerhalb eines strikt avantgardistischen Kunstverständnisses ist es schließlich die Postmoderne selbst, welche nicht etwa der Moderne in einem chronologischen Sinn folgt, sondern ihr sogar in einem scheinbar paradoxen Sinne vorausgeht! Oder in den Worten des philosophischen Meisterdenkers der Postmoderne: "Ein Werk ist nur modern, wenn es zuvor postmodern war. So gesehen bedeutet der Postmodernismus nicht das Ende des Modernismus, sondern den Zustand von dessen Geburt, und dieser Zustand ist konstant."[39] Diese auf den Gegensatz zwischen kanonisierter und noch nicht kanonisierter Kunst bezogene Umkehrung des Verhältnisses zwischen Moderne und Postmoderne macht nur allzu deutlich, was die Proklamation des "postmodernen Zeitalters" eigentlich beinhaltet: nämlich mitnichten einen neuen Epochenbegriff, sondern einen ironischen, in Form einer kunsttheoretischen Rexlexion vorgetragenen "Abschied" von den spezifischen Erfahrungsgehalten der ästhetischen Moderne, welche diese dabei allerdings nicht außer Kraft setzt, sondern in vielerlei Hinsicht sogar noch radikalisiert![40] Das einzig wirklich "Neue", das die Postmoderne beinhaltet, ist dabei die Entzauberung des Neuen selbst bzw. ein definitiver Abschied vom Modernismus im Sinne eines Avantgardismus, der sich seit seinen frühen Proklamationen um 1910 inzwischen bereits selbst historisch überholt hat und anstelle der schockartigen Inszenierung von vermeintlichen Traditionsbrüchen nun einem neuen Historismus Platz macht, in dem die für die Moderne schlechthin konstitutive Zeitdifferenz zwischen dem "Alten" und dem "Neuen" ihre normative Kraft verloren hat und inzwischen mithin selbst bereits einer Vergangenheit angehört, die gerade als Vergangenheit im post-modernen Kunstwerk zitierfähig geworden und aus diesem Grund selbst wiederum der Moderne bzw. der Gegenwart zuzurechnen ist.[41]

Insofern stellt die Postmoderne aber zugleich eine Form der Selbstbeschreibung und Selbstunterscheidung der Moderne dar, in der die Einheit von Selbstbezug und Fremdbezug als zentrale "Leitdifferenz" des modernen Kunstsystems gleichsam spielerisch zur Disposition gestellt worden ist und vermittels der die spezifische Souveränität bzw. Autonomie der Kunst innerhalb der modernen Gesellschaft eine eindrucksvolle Bestätigung findet. Denn offensichtlich besteht heute die gesellschaftliche Funktion der Kunst darin, das für die moderne Gesellschaft schlechthin grundlegende Prinzip der funktionalen Differenzierung zum Gegenstand ihrer eigenen kunsttheoretischen Selbstreflexion zu erheben und vermittels dieser Form der Unterscheidung den genuin gesellschaftlichen Charakter der Kunst selbst zu thematisieren.[42] Zugleich wird aber auch deutlich, daß gerade die in der "postmodern" gewordenen Kunst zum Ausdruck kommende Selbstunterscheidung der Moderne innerhalb der bildenden Kunst, Architektur und Literatur offensichtlich sehr unterschiedliche bereichsspezifische Grenzziehungen zwischen dem "Alten" und dem "Neuen" bzw. dem "Modernen" und dem "Postmodernen" zuläßt und sich insofern für den post-modernen Habitus übergreifende Kriterien ebenfalls nur noch in einem sehr formalen Sinn bzw. in einer spezifischen Form der Unterscheidung angeben lassen, welche die Möglichkeit von recht unterschiedlichen Beobachterstandpunkten keinesfalls ausschließt, sondern sie gerade eindrucksvoll bestätigt!

Die Entschleunigung des künstlerischen Wandels im Sinne einer nun selbst normativ gewendeten Gleichzeitigkeit des Ungleichzeitigen, die Überwindung des Gegensatzes zwischen elitärer Kultur und Massenkultur und die damit gegebene Möglichkeit einer Mehrsprachigkeit des post-modernen Kunstwerkes sowie die Aufhebung des für die "auratische" Kunst einstmals noch zentralen Gegensatzes zwischen Kunst und Leben, die gemeinhin mit dem Namen der "Postmoderne" verbunden werden, machen hierbei zum einen deutlich, daß die post-moderne Form der Selbstunterscheidung der Kunst die spezifisch esoterischen Erfahrungsgehalte der ursprünglich avantgardistisch verkündeten ästhetischen Moderne im Kunstsystem selbst frei verfügbar und als solche publikumswirksam darstellbar gemacht hat. Zum anderen können wir aus dieser Feststellung aber auch die Schlußfolgerung ziehen, daß in diesem Zusammenhang nun mit genuin ästhetischen Mitteln bzw.

Reflexionsformen eine Form der Selbstbeschreibung des Kunstsystems vorgenommen wird, die zugleich eine spezifische Differenz bzw. Eigentümlichkeit der modernen Gesellschaft schlechthin kennzeichnet: nämlich den 
Umstand, daß im Rahmen einer voll entfalteten funktionalen Differenzierung der einzelnen gesellschaftlichen Teilsysteme kein einzelnes von ihnen mehr den Anspruch erheben kann, eine adäquate, d.h. für alle übrigen Teilsysteme und die damit verbundenen alternativen Beobachterstandpunkte allgemeinverbindliche "Repräsentation der Gesellschaft in der Gesellschaft" darzustellen.[43] Gerade die Kunst bzw. die ästhetische Form der Selbstbeschreibung der modernen Gesellschaft macht deutlich, daß sich die Moderne selbst gar nicht mehr über eine historisch vorgegebene Tradition bzw. Vergangenheit definieren läßt, sondern nur noch durch eine ihr immanent zukommende Form der Unterscheidung, die sie im selben Atemzug zugleich wieder durchstreicht bzw. relativiert. Damit wird aber nicht nur der zutiefst kontingente Charakter jenes dem Prinzip der funktionalen Differenzierung zugrundeliegenden Aktes des Unterscheidens selbst deutlich, sondern auch eine spezifische Funktion der Kunst bzw. eine ihr eigentümliche Kunst der Unterscheidung, die sie von allen anderen gesellschaftlichen Teilsystemen abgrenzt und vermittels der sie ihre eigene autonome Stellung innerhalb der modernen Gesellschaft behauptet.[44]

Indem so die moderne Kunst in einer zunehmend paradoxer werdenen Form beständig mit ihren eigenen "Leitdifferenzen" wie der zwischen dem Alten und Neuen bzw. Kunst und Leben herumexperimentiert und sie inzwischen fast schon bis zur Ununterscheidbarkeit ineinander übergehen läßt, bestätigt sie aber nicht nur die spezifische Souveränität und den zutiefst kontingenten Charakter ihrer eigenen Unterscheidungs-Kunst, vermittels der sie sich von anderen gesellschaftlichen Teilsystemen abgrenzt, sondern zugleich auch eine fundamentale Erfahrung der Differenz, welche die Moderne schlechthin kennzeichnet: nämlich die, daß sie sich nicht mehr in einem genuin historischen Sinne begreifen läßt, sondern nur noch vermittels von Unterscheidungen, die ihrer eigenen Form der Selbstbeschreibung eigentümlich sind. Und gerade in dieser paradoxen Form der Aufhebung der Unterscheidung von Selbstbezug und Fremdbezug reflektiert sie zugleich ihre eigene epochale Differenz! Oder anders gesprochen: Der Versuch, die Moderne in verschiedene Phasen im Sinne von genuin historischen Stadien zu unterscheiden wie z.B. Früh-, Hoch-, Spät- oder gar Post-Moderne ist von Anfang an zu einem Scheitern verurteilt gewesen, da es sich im Falle der Moderne gar nicht mehr um eine Epoche in einem strikt historischen Sinne handelt, sondern um die paradoxe Grund-Form einer Unterscheidung zwischen dem "Alten" und dem "Neuen", welche die Moderne selbst vollzieht und die nur deshalb oft so schwer wiederzuerkennen ist, weil sie immer wieder in historisch verschiedenen Namen bzw. Kostümen vorgenommen wird. Insofern sind diese verschiedenen Selbstunterscheidungen der Moderne auch nicht mehr in einem strikt historischen Sinne zu verstehen, sondern nur noch als paradoxe Formen der Beschreibung der Einheit einer Leit-Differenz, die sie gerade epochal charakterisiert.[45]

Was bleibt also als Fazit? Sicher ist zumindest eines: Soviel Unterschied wie heute war noch nie! Und dies betrifft nicht nur die moderne Gesellschaft selbst oder gar ausschließlich nur ihr Kunstsystem, sondern zugleich auch jene Formen der disziplinären Beschreibung der Moderne, die seit dem 19. Jahrhundert unter dem KunstNamen der Soziologie zusammengefaßt worden sind. Deren eigene Kunst der Unterscheidung bestünde dann aber in Zukunft darin, nicht mehr den vermeintlich ganzheitlichen und insofern notwendig gescheiterten traditionellen Beschreibungsformen der modernen Gesellschaft nachzutrauern, sondern sich ebenfalls ohne nostalgischen Rückblick auf die eigene disziplinäre Vergangenheit bzw. die damit notwendig verbunden gewesenen Illusionen in einem selbstbewußten und zugleich konstruktiven Sinne an jenem spezifisch zeitgenössischen Gesellschaftsspiel zu beteiligen, in dem die Soziologie nun selbst ohne Trauer über den "Verlust der Mitte" bzw. eines vermeintlich privilegierten Beobachterstandpunktes ihre eigenen Formen der Unterscheidung gegenüber konkurrierenden Beschreibungen der Moderne geltend zu machen, aber zugleich auch zu bewähren hat.[46]

In: Johannes Weiß (Hrsg.), Mehrdeutigkeiten der Moderne (= Intervalle 1. Schriften zur Kulturforschung). Kassel: Kassel University Press 1998, S. 43-87 
Klaus Lichtblau, geb. 1951 in Karlsruhe, studierte Philosophie, Politikwissenschaft und Soziologie in München und Bielefeld. Promotion an der Fakultät für Geschichtswissenschaft und Philosophie der Universität Bielefeld. Habilitation am Fachbereich Gesellschaftswissenschaften der Universität Kassel. 1975-1980 wissenschaftlicher Mitarbeiter in der Redaktion des Historischen Wörterbuchs der Philosophie in Bielefeld. 1981-1983 Forschungsstipendiat der Fritz Thyssen Stiftung und Lehrbeauftragter an der Universität Bielefeld. 1984-1988 verantwortlicher Redakteur der Besprechungszeitschrift Soziologische Revue. 1989-1995 wissenschaftlicher Assistent am Fachbereich Gesellschaftswissenschaften der Universität Kassel. Zur Zeit Lehrstuhlvertreter am Institut für Soziologie die Christian-Albrechts-Universität zu Kiel. Wichtigste Buchveröffentlichungen: Theorie der bürgerlichen Gesellschaft. Zum Verhältnis von Ökonomie, Recht und Politik (Gießen 1978); Kulturkrise und Soziologie um die Jahrhundertwende. Zur Genealogie der Kultursoziologie in Deutschland (Frankfurt am Main 1996); Georg Simmel (Frankfurt am Main 1997). Mitherausgeber von: Feministische Vernunftkritik. Ansätze und Traditionen (Frankfurt am Main 1992); Max Weber, Die protestantische Ethik und der "Geist" des Kapitalismus (Bodenheim 1993); Max-Weber-Symposium in Erfurt (Berlin 1995). Zahlreiche weitere Veröffentlichungen in Fachzeitschriften und Sammelwerken auf dem Gebiet der soziologischen Theorie und ihrer Geschichte sowie der Kultur- und Sozialphilosophie.

Der vorliegende Essay ist die ausgearbeitete Fassung des Vortrages, den ich anläßlich meines Habilitationsverfahrens am 14. Februar 1996 vor dem Habilitationsgremium des Fachbereichs Gesellschaftswissenschaften der Universität Kassel gehalten habe.

[2] Dieses unter den Bedingungen der Moderne als solches nicht mehr aufhebbare Spannungsverhältnis zwischen Einheit und Vielheit kennzeichnete bekanntlich noch die Mannheimsche Form der modernen Wissenssoziologie, die zumindest bezüglich der dabei in Aussicht gestellten Möglichkeit einer "neuen Kultursynthese" notwendig scheitern mußte. Vgl. Klaus Lichtblau, Auf der Suche nach einer neuen Kultursynthese. Zur Genealogie der Wissenssoziologie Max Schelers und Karl Mannheims, in: Sociologia Internationalis 30 (1992), S. 1-33.

[3] Siehe hierzu auch Klaus Lichtblau, Soziologie und Zeitdiagnose. Oder: Die Moderne im Selbstbezug, in: Stefan Müller-Doohm, Jenseits der Utopie. Theoriekritik der Gegenwart, Frankfurt am Main: Suhrkamp 1991, S. $15-47$

[4] Vgl. Werner Sombart, Der moderne Kapitalismus. Historisch-systematische Darstellung des gesamteuropäischen Wirtschaftslebens von seinen Anfängen bis zur Gegenwart, Bd. III: Das Wirtschaftsleben im Zeitalter des Hochkapitalismus, München/Leipzig: Duncker \& Humblot 1927, 2. Halbband, S. 1008 ff.; ders., Die Wandlungen des Kapitalismus, in: Verhandlungen des Vereins für Sozialpolitik in Zürich 13. bis 15. September 1928, München/Leipzig: Duncker \& Humblot 1929, S. 23-41; ders., Die Zukunft des Kapitalismus, BerlinCharlottenburg: Buchholz \& Weisswange 1932; ders., Deutscher Sozialismus, ebd. 1934; ferner Friedrich Lenger, Werner Sombart 1863-1941. Eine Biographie, München 1994, S. 332 ff.

[5] Zu den mit der Erforschung der Frühen Neuzeit verbundenen Problemen einer eindeutigen Periodisierung dieses dabei als Einheit unterstellten "neuen Zeitalters" siehe auch Wilhelm Kamlah, "Zeitalter" überhaupt, "Neuzeit" und "Frühneuzeit", in: Saeculum 8 (1957), S. 313-332; Ernst Walder, Zur Geschichte und Problematik des Epochenbegriffs "Neuzeit" und zum Problem der Periodisierung der europäischen Geschichte, in: ders. u.a. (Hrsg.), Festgabe Hans von Greyerz zum sechzigsten Geburtstag 5. April 1967, Bern: Lang 1967, S. 21-47; Stephan Skalweit, Der Beginn der Neuzeit. Epochengrenze und Epochenbegriff, Darmstadt: Wissenschaftliche Buchgesellschaft 1982; Horst Günther, Art. "Neuzeit, Mittelalter, Altertum", in: Joachim Ritter/Karlfried Gründer (Hrsg.), Historisches Wörterbuch der Philosophie, Bd. 6, Basel: Schwabe \& Co. 1984, Sp. 782-798; Reinhart Koselleck, Das achtzehnte Jahrhundert als Beginn der Neuzeit, in: Reinhart Herzog/Reinhart Koselleck (Hrsg.), 
Epochenschwelle und Epochenbewußtsein, München: Fink 1987, S. 269-282; ferner Rudolf Vierhaus (Hrsg.), Frühe Neuzeit - Frühe Moderne? Forschungen zur Vielschichtigkeit von Übergangsprozessen, Göttingen: Vandenhoeck \& Ruprecht 1992.

[6] Vgl. Zygmunt Bauman, Modernity and Ambivalence, Cambridge: Polity Press 1991; ders., Intimations of Postmodernity, London: Routledge 1991; ferner Wolfgang Bonß, Die uneindeutige Moderne. Anmerkungen zu Zygmunt Bauman, in: Mittelweg 36, Jg. 2 (1993), Heft 4, S. 1-10.

[7] Zu dieser nicht nur für die ältere wissenssoziologische Tradition, sondern auch für die zeitgenössische soziologische Forschung geradezu konstitutiven "Leitdifferenz" zwischen Gesellschaft und Kultur bzw. Sozialstruktur und Semantik siehe auch Talcott Parsons, Culture and Social System Revisited, in: Social Science Quarterly 53 (1972), S. 253-266; Karl-Siegbert Rehberg, Kultur versus Gesellschaft? Anmerkungen zu einer Streitfrage in der deutschen Soziologie, in: Friedhelm Neidhardt/M. Rainer Lepsius/Johannes Weiß (Hrsg.), Kultur und Gesellschaft (= Kölner Zeitschrift für Soziologie und Sozialpsychologie, Sonderheft 27), Opladen: Westdeutscher Verlag 1986 S. 92-115; Friedrich Tenbruck, Die kulturellen Grundlagen der Gesellschaft. Der Fall der Moderne, Opladen: Westdeutscher Verlag 1989, bes. S. 80 ff. u. 251 ff.; Niklas Luhmann,

Gesellschaftsstruktur und Semantik. Studien zur Wissenssoziologie der modernen Gesellschaft, 4 Bde., Frankfurt am Main: Suhrkamp 1980-1995, (hier besonders der programmatische Aufsatz "Gesellschaftliche Struktur und semantische Tradition" im ersten Band auf S. 9-71); ferner die entsprechenden Überlegungen bei Alois Hahn, Funktionale und stratifikatorische Differenzierung und ihre Rolle für die gepflegte Semantik. Zu Niklas Luhmanns "Gesellschaftsstruktur und Semantik", in: Kölner Zeitschrift für Soziologie und Sozialpsychologie 33 (1981), S. 345-360.

[8] Vgl. hierzu die stark kanonische Züge tragende und insofern durchaus repräsentative Zusammenfassung dieses orthodoxen soziologischen Modernitätsverständnisses bei Johannes Berger, Modernitätsbegriffe und Modernitätskritik in der Soziologie, in: Soziale Welt 39 (1988), S. 224-236 (die "Zäsur welthistorischen Ausmaßes" findet dort auf S. 225 statt). Zur philosophischen Diskussion dieses spezifischen Erfahrungsgehaltes der "Neuzeit" bzw. der "Moderne" siehe auch Hans Blumenberg, Die Legitimität der Neuzeit, 2. Aufl. Frankfurt am Main: Suhrkamp 1988 sowie Stephen Toulmin, Kosmopolis. Die unerkannten Aufgaben der Moderne, Frankfurt am Main: Suhrkamp 1991.

[9] Vgl. Max Weber, Die protestantische Ethik und der "Geist" des Kapitalismus. Textausgabe auf der Grundlage der ersten Fassung von 1904/05 mit einem Verzeichnis der wichtigsten Zusätze und Veränderungen aus der zweiten Fassung von 1920 hrsg. u. eingel. von Klaus Lichtblau u. Johannes Weiß, Bodenheim: Athenäum/ Hain/Hanstein 1993, S. 32 f.; ferner ders., Gesammelte Aufsätze zur Religionssoziologie, Bd. 1, Tübingen: Mohr 1920, S. 11 f. u. 265 f. Zur neueren soziologischen Theorie der funktionalen Differenzierung der modernen Gesellschaft siehe auch Hartmann Tyrell, Anfragen an die Theorie der gesellschaftlichen Differenzierung, in: Zeitschrift für Soziologie 7 (1978), S. 175-193; Renate Mayntz u.a., Differenzierung und Verselbständigung. Zur Entwicklung gesellschaftlicher Teilsysteme, Frankfurt am Main/New York: Campus 1988; Hans van der Loo/ Willem van Reijen, Modernisierung. Projekt und Paradox, München: DTV 1992, S. 81 ff.; Jeffrey C. Alexander, Soziale Differenzierung und kultureller Wandel. Studien zur neofunktionalistischen Gesellschaftstheorie, Frankfurt am Main/New York: Campus 1993; ferner Uwe Schimank, Theorien gesellschaftlicher Differenzierung, Opladen: Leske + Budrich 1996.

[10] Siehe hierzu insbesondere den programmatischen Aufsatz von Reinhart Koselleck, >Neuzeit $<$. Zur Semantik moderner Bewegungsbegriffe, in: ders. (Hrsg.) Studien zum Beginn der modernen Welt, Stuttgart: KlettCotta 1977, S. 264-299; ferner die bereits zitierten wissenssoziologischen Arbeiten von Niklas Luhmann.

[11] Zur epochalen Standortbestimmung und ausführlichen Diskussion dieser überhaupt erst auf dem Boden der "neuzeitlich bewegten Geschichte" möglich gewordenen "alteuropäischen" Form der Kulturkritik siehe Hans Robert Jauß, Der literarische Prozeß des Modernismus von Rousseau bis Adorno, in: Ludwig von Friedeburg/ Jürgen Habermas (Hrsg.), Adorno-Konferenz 1983, Frankfurt am Main: Suhrkamp 1983, S. 95-130; Jürgen Habermas, Der philosophische Diskurs der Moderne. Zwölf Vorlesungen, Frankfurt am Main: Suhrkamp 1985; 
ferner Cornelia Klinger, Flucht - Trost - Revolte. Die Moderne und ihre ästhetischen Gegenwelten, München/ Wien: Hanser 1995.

[12] Vgl. Ferdinand Tönnies, Gemeinschaft und Gesellschaft. Abhandlung des Communismus und des Socialismus als empirischer Culturformen, Leipzig: Reisland 1887, S. 45 ff.; ders., Gemeinschaft und Gesellschaft, in: Alfred Vierkandt (Hrsg.), Handwörterbuch der Soziologie, Stuttgart: Enke 1931, S. 180-191 sowie ders., Die Entstehung meiner Begriffe Gemeinschaft und Gesellschaft, in: Kölner Zeitschrift für Soziologie und Sozialpsychologie 7 (1955), S. 463-467. Zur Diskussion des tieferen Bedeutungsgehaltes dieses das ganze Tönniessche Werk durchziehenden grundbegrifflichen Gegensatzpaares siehe ferner René König, Die Begriffe Gemeinschaft und Gesellschaft bei Ferdinand Tönnies, in: Kölner Zeitschrift für Soziologie und Sozialpsychologie 7 (1955), S. 348-420.

[13] Zur Eigenart von Max Webers "Entwicklungsgeschichte" des okzidentalen Rationalismus siehe insbesondere den einschlägigen Aufsatz von Guenther Roth, Max Webers Entwicklungsgeschichte und historische Soziologie, in: ders., Politische Herrschaft und persönliche Freiheit. Heidelberger Max WeberVorlesungen 1983, Frankfurt am Main: Suhrkamp 1987, S. 283-305. Die Komplexität des in Webers historischer Soziologie zum Ausdruck kommenden universalgeschichtlichen Rationalisierungsprozesses wird auch in den neueren Untersuchungen von Stephen Kalberg deutlich. Vgl. Kalberg, Max Webers historisch-vergleichende Untersuchungen und das "Webersche Bild der Neuzeit": Eine Gegenüberstellung, in: Johannes Weiß (Hrsg.), Max Weber heute. Erträge und Probleme der Forschung, Frankfurt am Main: Suhrkamp 1989, S. 425-444 sowie ders., Max Weber's Comparative-Historical Sociology, Cambridge: Polity Press 1994. Zu der dadurch bewirkten Veränderung der historisch-politischen Semantik in der europäischen Neuzeit siehe auch die von Reinhart Koselleck verfaßte Einleitung zu Otto Brunner/Werner Conze/Reinhart Koselleck (Hrsg.), Geschichtliche Grundbegriffe. Historisches Lexikon zur politisch-sozialen Sprache in Deutschland, Bd. 1, Stuttgart: Klett-Cotta 1972, S. XIII-XXVII.

[14] Vgl. Georg Simmel, Philosophische Kultur. Über das Abenteuer, die Geschlechter und die Krise der Moderne. Gesammelte Essais (1911). Mit einem Nachwort von Jürgen Habermas, Berlin 1983, S. 152. Zur ausführlichen Analyse und Diskussion von Simmels Verständnis der ästhetischen Moderne siehe David Frisby, Sociological Impressionism. A Reassessment of Georg Simmel`s Social Theory, London: Routledge 1981, bes. S. 102 ff.; ders., Fragments of Modernity. Theories of Modernity in the Work of Simmel, Kracauer and Benjamin, Cambridge: Polity Press 1985, S. 1-108; ferner ders., Simmel and Since. Essays on Georg Simmel`s Social Theory, London/New York: Routledge 1992, S. 64 ff. u. 135 ff. Siehe hierzu auch die entsprechenden Ausführungen in Klaus Lichtblau, Kulturkrise und Soziologie um die Jahrhundertwende. Zur Genealogie der Kultursoziologie in Deutschland, Frankfurt am Main: Surhkamp 1996, S. 203 ff.

[15] Vgl. Max Weber, Gesammelte Aufsätze zur Religionssoziologie, Bd. I, a.a.O., S. 554 ff. sowie die entsprechende grundbegriffliche Unterscheidung zwischen "Rationalisierung" und "Sublimierung" bei Howard L. Kaye, Rationalization as Sublimation: On the Cultural Analysis of Weber and Freud, in: Theory, Culture \& Society, Jg. 9 (1992), Heft 4, S. 45-74. Dieser vor dem Hintergrund von Webers Freud-Lektüre zu sehende Begriff der "Sublimierung" von genuin ästhetischen und erotischen Erfahrungsgehalten entspricht im übrigen sachlich genau dem, was Georg Simmel am Beispiel der "modernen Seele" sowie des Geldes als einen Prozeß der Läuterung im Sinne einer immer reinlicheren Scheidung von vormals miteinander bis zur Ununterscheidbarkeit vermengten Sinnsphären beschrieben hatte. Siehe hierzu auch die entsprechenden Ausführungen bei Hannes Böhringer, Spuren von spekulativem Atomismus in Simmels formaler Soziologie, in: Hannes Böhringer/Karlfried Gründer (Hrsg.), Ästhetik und Soziologie um die Jahrhundertwende: Georg Simmel, Frankfurt am Main: Klostermann 1976, S. 105-117; ferner Klaus Lichtblau, Die Seele und das Geld. Kulturtheoretische Implikationen in Georg Simmels "Philosophie des Geldes", in: Friedhelm Neidhardt/M. Rainer Lepsius/Johannes Weiß (Hrsg.), Kultur und Gesellschaft (= Kölner Zeitschrift für Soziologie und Sozialpsychologie, Sonderheft 27), Opladen: Westdeutscher Verlag 1986, S. 57-74. Zu den mit dieser grundbegrifflichen Unterscheidung verbundenen Antinomien der Moderne siehe auch Johannes Weiß, Vernunft und Vernichtung. Zur Philosophie und Soziologie der Moderne, Opladen: Westdeutscher Verlag 1993, S. 148 ff. 
[16] Zu diesem spezifisch normativen Gehalt der älteren Variante der sozialwissenschaftlichen

Modernisierungs-theorie vgl. z.B. die einschlägigen Arbeiten von Talcott Parsons, Evolutionary Universals in Society, in: American Sociological Review 29 (1964), S. 339-357; ders., Societies: Evolutionary and Comparative Perspectives, Englewood Cliffs: Prentice Hall 1966; ders., Comparative Studies and Evolutionary Change, in: Ivan Vallier (Hrsg.), Comparative Methods in Sociology, Berkeley: University of California Press 1971, S. 97-139; Reinhard Bendix, Tradition and Modernity Reconsidered, in: Comparative Studies in Society and History 9 (1967), S. 292-346; ferner Wolfgang Zapf, Die soziologische Theorie der Modernisierung, in: Soziale Welt 26 (1975), S. 212-226. Zur ausführlichen Diskussion der fachlichen und politischen Implikationen dieser Variante der sozialwissenschaftlichen Modernisierungs-theorie siehe ferner M. Rainer Lepsius, Soziologische Theoreme über die Sozialstruktur der "Moderne" und die "Modernisierung", in: Reinhart Koselleck (Hrsg.), Studien zum Beginn der modernen Welt, a.a.O., S. 10-29; Anton Sterbling, Modernisierung und soziologisches Denken. Analysen und Betrachtungen, Hamburg: Krämer 1991; ferner Peter Wehling, Die Moderne als Sozialmythos. Zur Kritik sozialwissenschaftlicher Modernisierungstheorien, Frankfurt am Main/ New York: Campus 1992.

[17] Diese Revision von zentralen Grundannahmen der "orthodoxen" Modernisierungsforschung wird zum Beispiel in den neueren Arbeiten von Shmuel N. Eisenstadt sowie Wolfgang Zapf deutlich. Vgl. Eisenstadt, Tradition, Wandel und Modernität, Frankfurt am Main: Suhrkamp 1979; ders., Die Antinomien der Moderne. Die jakobinischen Grundzüge der Moderne und des Fundamentalismus. Heterodoxien, Utopismus und Jakobinismus in der Konstitution fundamentalistischer Bewegungen, Frankfurt am Main: Suhrkamp 1997 (im Erscheinen); ferner Zapf, Modernisierung, Wohlfahrtsentwicklung und Transformation. Soziologische Aufsätze 1987-1994, Berlin: Edition Sigma 1994.

[18] Vgl. Daniel Bell, The Cultural Contradictions of Capitalism, New York: Basic Books 1976, bes. S. 33 ff.; ders., Beyond Modernism, beyond Self, in: Quentin Anderson u.a. (Hrsg.), Art, Politics and Will. Essays in Honour of Lionel Trilling, New York: Basic Books 1977, S. 213-253; ders., Kapitalismus und Kultur. Vom Ende des Modernismus, in: Der Monat, NF 288 (1983), S. 158-172; ferner ders., Zur Auflösung der Wiederprüche von Modernität und Modernismus: Das Beispiel Amerikas, in: Heinrich Meier (Hrsg.), Zur Diagnose der Moderne, München/Zürich: Piper 1990, S. 21-67.

[19] Siehe hierzu insbesondere den bereits "klassischen" Aufsatz von Walter Benjamin, Das Kunstwerk im Zeitalter seiner technischen Reproduzierbarkeit (1936), in: ders., Gesammelte Schriften. Werkausgabe, Bd. 2, Frankfurt am Main: Suhrkamp 1980, S. 431-508; ferner Theodor W. Adorno, Ästhetische Theorie, hrsg. v. Gretel Adorno u. Rolf Tiedemann, Frankfurt am Main: Suhrkamp 1970, bes. S. 9 ff., 56 ff., 122 ff., 262 ff. u. 491 ff. [20] Vgl. Pierre Bourdieu, Die feinen Unterschiede. Kritik der gesellschaftlichen Urteilskraft, Frankfurt am Main: Suhrkamp sowie 1982; ders., Les règles de l'art. Genèse et structure du champs littéraire, Paris: Editions du Seuil 1992; Michel Maffesoli, Das ästhetische Paradigma. Soziologie als Kunst, in: Soziale Welt 38 (1987), S. 460-470; ders., Au creux des apparences. Pour une éthique de l'esthétique, Paris: Plon 1990; Karla Fohrbeck, Kunst als Produktivfaktor oder Der Überbau als Basis, in: Ästhetik und Kommunikation 67-68 (1987), S. 91-93; Mike Featherstone, Consumer Culture \& Postmodernism, London: Sage 1991, bes. S. 65 ff.; Gerhard Schulze, Die Erlebnisgesellschaft. Kultursoziologie der Gegenwart, Frankfurt am Main/New York: Campus 1992; Scott Lash, Ästhetische Dimensionen reflexiver Modernisierung, in: Soziale Welt 43 (1992), S. 261-277; ferner Dorothea S. Whitten/Norman E. Whitten Jr., Imagery and Creativity. Ethnoaesthetics and Art Worlds in the Americas, Tucson: University of Arizona Press 1993.

Vgl. Peter L. Berger, A Rumor of Angels. Modern Society and the Rediscovery of the Supranatural, New York: Doubleday 1969; ders., The Heretical Imperative. Contemporary Possibilities of Religious Affirmation, New York: Doubleday 1979; ders., Sehnsucht nach Sinn. Glauben in einer Zeit der Leichtgläubigkeit, Frankfurt am Main/New York: Campus 1994; Robert N. Bellah, Beyond Belief. Essays on Religion in a Post-Traditional World, New York: Harper \& Row 1970; Robert Bellah/Charles Glock (Hrsg.), The New Religious Consciousness, Berkeley: University of California Press 1976; R. Bellah u.a., Habits of the Heart. Individualism 
and Commitment in American Liefe, Berkeley/Los Angeles: University of California Press 1985; Daniel Bell, The Return of the Sacred? The Argument on the Future of Religion, in: ders., The Winding Passage. Essays and Sociological Journeys 1960-1980, Cambridge, MA: ABT Books 1980, S. 324-354; ders., The Cultural Contradictions of Capitalism, a.a.O., S. 146 ff.; Robert Wuthnow, Experimentation in American Religion. The New Mysticism \& Their Implications for the Churches, Berkeley/Los Angeles: University of California Press 1978; ders., The Restructuring of American Religion, Princeton: Princeton University Press 1988; Mary Douglas/ Steven Tipton (Hrsg.), Religion and America. Spiritual Life in a Secular Age, Boston: Beacon Press 1982; Thomas Robbins, Cults, Converts and Charisma. The Sociology of New Religious Movements, London: Sage 1988. Siehe ferner die entsprechenden Beiträge in Jörg Bergmann/Alois Hahn/Thomas Luckmann (Hrsg.), Religion und Kultur (= Kölner Zeitschrift für Soziologie und Sozialpsychologie, Sonderheft 33), Opladen: Westdeutscher Verlag 1993.

[22] Vgl. Jürgen Habermas, Die Moderne - ein unvollendetes Projekt (1980), in: ders., Kleine Politische Schriften (I-IV), Frankfurt am Main: Suhrkamp 1981, S. 444-464 (hiers bes. S. 457 ff.). Bezüglich dem spezifisch surrealistischen Versuch der Aufhebung von Kunst in Lebenspraxis siehe auch Karlheinz Barck (Hrsg.), Surrealismus in Paris 1919-1939. Ein Lesebuch, Leipzig: Reclam 1990.

[23] Adorno, Ästhetische Theorie, a.a.O., S. 57.

[24] Vgl. Habermas, Die Moderne - ein unvollendetes Projekt, a.a.O.; ferner ders., Theorie des kommunikativen Handelns, 2 Bde., Frankfurt am Main: Suhrkamp 1981, bes. Bd. 2, S. 548 ff.

[25] Vgl. hierzu insbesondere Niklas Luhmann, Soziale Systeme. Grundriß einer allgemeinen Theorie, Frankfurt am Main: Suhrkamp 1984; ferner ders., Beobachtungen der Moderne, Wiesbaden: Westdeutscher Verlag 1992, bes. S. 11-49. Zu einer solchen Form der "autopoietischen" Reformulierung der modernen sozialwissenschaftlichen Systemtheorie siehe auch Danilo Zolo, Reflexive Selbstbegründung der Soziologie und Autopoiesis. Über die epistemologischen Voraussetzungen der "allgemeinen Theorie sozialer Systeme" Niklas Luhmanns, in: Soziale Welt 36 (1985), S. 483-502.

[26] Zur ausführlichen Rekonstruktion der Marxschen Form der Analyse des modernen kapitalistischen Wirtschaftssystems als einem autonom gewordenen ökonomischen System der Produktion und Reproduktion, das gleichsam "Autopoiesis" in einem einzigen Fall betreibt, der gleichwohl für das Ganze stehen soll, siehe auch Klaus Lichtblau, Theorie der bürgerlichen Gesellschaft. Zum Verhältnis von Ökonomie, Recht und Politik, Gießen: Focus 1978, bes. S. 227 ff.

[27] Vgl. Ulrich Beck, Risikogesellschaft. Auf dem Weg in eine andere Moderne, Frankfurt am Main: Suhrkamp 1986; ders., Die Frage nach der anderen Moderne, in: Deutsche Zeitschrift für Philosophie 39 (1991), S. 1297-1308 sowie ders., Die Erfindung des Politischen. Zu einer Theorie reflexiver Modernisierung, Frankfurt am Main: Suhrkamp 1993.

[28] Beck, Risikogesellschaft, a.a.O., S. 20. Vgl. auch ders., Der clevere Bürger. Bemerkungen zu Anthony Giddens' Konzeption "reflexiver Modernisierung", in: Soziologische Revue 19 (1996), S. 3-9.

[29] Dieses bereits seiner Marburger Antrittsvorlesung vom 10. November 1995 über die "Konturen einer Soziologie für das 21. Jahrhundert" zugrundeliegende Mißverständnis von Soziologie bzw. einer damit verbundenen professionspolitischen "Einmischung" in die Händel dieser Welt hat Dirk Käsler erst kürzlich wieder anläßlich seiner eigenen Einmischung in jene die ganze Fachgeschichte seit der Jahrhundertwende prägende und neuerdings von Wanfried Dettling wieder angemahnte Diskussion über den Sinn bzw. Unsinn eines eigenständigen akademischen Fachs Soziologie medienwirksam zum Ausdruck gebracht. Vgl. Warnfried Dettling, Fach ohne Boden. Brauchen wir überhaupt noch Soziologen?, in: Die Zeit, Nr. 2, 5. Januar 1996, S. 23 sowie Dirk Käsler, Suche nach der guten Gesellschaft, in: Die Zeit, Nr. 4, 19. Januar 1996, S. 43. Leider hat Käsler aber bei seinem Versuch, der Soziologie wieder den Status einer "Moralwissenschaft" anzuempfehlen, das Kind gleichsam mit dem Bade ausgeschüttet, da er trotz der erst kürzlich erfolgten Neuauflage seines bewährten Einführungswerkes zu Max Weber heute offensichtlich selbst nicht mehr in der Lage ist, die beiden Extreme innerhalb der von Max Weber konsequent durchgeführten Unterscheidung zwischen einer theoretischen 
Wertbeziehung und einer praktisch-wertenden Stellungnahme strikt auseinanderzuhalten. Denn eine "wertende Soziologie" in dem von Käsler heute favorisierten Sinne war für Max Weber bekanntlich das rote Tuch schlechthin, bei dem er nun wirklich keinen Spaß mehr verstand, wie der unter seiner Beteiligung geprägte Verlauf der entsprechenden Diskussionen im Verein für Socialpolitik sowie auf den ersten beiden Soziologentagen von 1910 und 1912 eindrucksvoll belegt! Siehe hierzu auch die entsprechenden Ausführungen bei Dieter Lindenlaub, Richtungskämpfe im Verein für Sozialpolitik 1890-1914, 2 Bde., Wiesbaden: Steiner 1967 sowie Ursula Karger, Institutionsgeschichtliche Zäsuren in der deutschen Soziologie, Dissertation Universität Bochum 1978.

[30] Vgl. Anthony Giddens, The Consequences of Modernity, Cambridge: Polity Press 1990; ders., Modernity and Self-Identity. Self and Society in the Late Modern Age, Cambridge: Polity Press 1991; ders., Tradition in der post-traditionalen Gesellschaft, in: Soziale Welt 44 (1993), S. 445-485 (hier bes. S. 483 ff.); ferner ders., Beyond Left and Right. The Future of Radical Politics, Cambridge: Polity Press 1994.

[31] Lash, Ästhetische Dimensionen reflexiver Modernisierung, a.a.O., S. 262; zur weiterführenden Diskussion der mit einer solchen kulturtheoretischen Radikalisierung unseres Modernitätsverständnisses verbundenen Implikationen siehe auch das nun als Teamwork erschienene Buch von Ulrich Beck/Anthony Giddens/Scott Lash, Reflexive Modernization. Politics, Tradition and Aesthetics in the Modern Social Order, Cambridge: Polity Press 1994.

[32] Vgl. hierzu und zum folgenden Walter Freund, Modernus und andere Zeitbegriffe des Mittelalters, Köln: Böhlau 1957; Elisabeth Gössmann, Antiqui und Moderni im Mittelalter. Eine geschichtliche Standortbestimmung, München: Schöningh 1974; Albert Zimmermann (Hrsg.), Antiqui und Moderni. Traditionsbewußtsein im späten Mittelalter, Berlin/New York: de Gruyter 1974. Siehe ferner auch die einschlägigen Übersichtsartikel von Fritz Martini, Art. "Modern, die Moderne", in: Werner Kohlschmidt/ Wolfgang Mohr (Hrsg.), Reallexikon der deutschen Literaturgeschichte, 2. Aufl., Bd. 2, Berlin: de Gruyter 1965, S. 391-415; Hans Robert Jauß, Literarische Tradition und gegenwärtiges Bewußtsein der Modernität. Wortgeschichtliche Betrachtungen, in: Hans Steffen (Hrsg.), Aspekte der Modernität, Göttingen: Vandenhoeck \& Ruprecht 1965, S. 150-197; ders., Art. "Antiqui/moderni (Querelle des Anciens et des Modernes)", in: Joachim Ritter/Karlfried Gründer (Hrsg.), Historisches Wörterbuch der Philosophie, Bd. 1, Basel: Schwabe \& Co. 1971, Sp. 410-414; Jost Schneider, Ein Beitrag zu dem Problem der "Modernität", in: Der Deutschunterricht 23 (1971), Heft 6, S. 58-67; ferner Hans Ulrich Gumbrecht, Art. "Modern, Modernität, Moderne", in: Otto Brunner/Werner Conze/Reinhart Koselleck (Hrsg.), Geschichtliche Grundbegriffe. Historisches Lexikon zur politisch-sozialen Sprache in Deutschland, Bd. 4, Stuttgart: Klett-Cotta 1978, S. 93-131.

[33] Diesem Umstand verdankt sich im übrigen auch die Prägung des von Bernhard von Chartres stammenden berühmten Bildes von den Zwergen, die bekanntlich auf den Schultern der Riesen sitzen und gerade deshalb eben doch ein klein wenig weiter schauen können als ihre antiken Vorgänger! Siehe hierzu auch die instruktive wissenssoziologische Studie von Robert K. Merton, Auf den Schultern von Riesen. Ein Leitfaden durch das Labyrinth der Gelehrsamkeit, Frankfurt am Main: Syndikat 1980.

[34] Vgl. hierzu die einschlägigen Studien von Hans Robert Jauß, Ästhetische Normen und geschichtliche Reflexion in der $>$ Querelle des Anciens et des Modernes $<$, in: Charles Perrault, Parallèle des anciens et des modernes en ce qui regarde les arts et les sciences (1688-1697), Neudruck München: Eidos 1964, S. 8-64; ders., Ursprung und Bedeutung der Fortschrittsidee in der "Querelle des Anciens et des Modernes", in: Helmut Kuhn/ Franz Wiedemann (Hrsg.), Die Philosophie und die Frage nach dem Fortschritt, München: Pustet 1964, S. 51-72; Werner Krauss, Der Streit der Altertumsfreunde mit den Anhängern der Moderne und die Entstehung des geschichtlichen Weltbildes, in: Werner Krauss/Hans Kortum (Hrsg.), Antike und Moderne in der Literaturdiskussion des 18. Jahrhunderts, Berlin: Akademie Verlag 1966, S. IX-LX; Hans Kortum, Die Hintergründe einer Akademiesitzung im Jahre 1687, ebd., S. LXI-CXI; ders., Charles Perrault und Nicolas Boileau, Berlin: Rütten \& Loening 1966; ferner Hartmut Stenzel, Die französische $>$ Klassik $<$. Literarische Modernisierung und absolutistischer Staat, Darmstadt: Wissenschaftliche Buchgesellschaft 1995. 
Dies wird besonders deutlich in dem berühmten Diktum von La Bruyère aus dem Jahre 1688: "Nous, qui sommes si modernes, serons anciens dans quelque siècles". Vgl. Jean de la Bruyère, Discours sur Théophraste (1688), in: ders., Les caractères, ou le moeurs de ce siècle (Einleitung). Oeuvres complètes, nouvelle éd. Paris: Julien Benda 1951, S. 3-18 (hier S. 11).

[36] Dies ist denn auch der eigentliche differenzierungstheoretische Ausgangspunkt der neueren kunstsoziologischen Analysen von Niklas Luhmann, denen ich mich im folgenden weitgehend anschließe. Vgl. Luhmann, Die Evolution des Kunstsystems, in: Kunstforum 124 (1993), S. 221-228 (zur "Aufsprengung des alteuropäischen Rationalitätskontinuums" vgl. dort S. 226); ders., Die Ausdifferenzierung des Kunstsystems, Bern: Benteli 1994 sowie ders., Die Kunst der Gesellschaft, Frankfurt am Main: Suhrkamp 1995. Gerd Hemmerich hat in diesem Zusammenhang hervorgehoben, daß das als autonome ästhetische Sphäre ausdifferenzierte moderne Kunstsystem aber seinerseits nur eine "translatio autonomiae" der neuzeitlichen Autonomie der Wissenschaften darstelle und insofern Wissenschaft und Kunst unter diesem spezifischen Gesichtspunkt trotz ihrer jeweiligen "Eigensinnigkeit" dennoch wieder miteinander vergleichbar sind. Vgl. Hemmerich, Überlegungen zum Phänomen der Moderne und ihrer Geschichte, in: Theo Elm/Gerd Hemmerich (Hrsg.), Zur Geschichtlichkeit der Moderne. Der Begriff der literarischen Moderne in Theorie und Deutung, München: Fink 1982, S. 23-41.

[37] Siehe hierzu die entsprechenden Ausführungen bei Hans Egon Holthusen, Kunst und Revolution, in: Bayerische Akademie der Schönen Künste (Hrsg.), Avantgarde. Geschichte und Krise einer Idee, München: Oldenbourg 1966, S. 7-44; Herbert Anton, Modernität als Aporie und Ereignis, in: Hans Steffen (Hrsg.), Aspekte der Modernität, a.a.O., S. 7-30; Jauß, Literarische Tradition und gegenwärtiges Bewußtsein der Modernität, a.a. O., S. 182 ff.; ders., Der literarische Prozeß des Modernismus von Rousseau bis Adorno, a.a.O., S. 113 ff.; Schneider, Ein Beitrag zum Problem der >Modernität<, a.a.O., S. 64 ff.; Gumbrecht, Art. "Modern, Modernität, Moderne", a.a.O., S. $114 \mathrm{ff}$.

[38] Vgl. James Ackerman, The Demise of the Avant Garde. Notes on the Sociology of Recent American Art, in: Comparative Studies in Society and History 11 (1969), S. 371-384; Peter Bürger, Theorie der Avantgarde, Frankfurt am Main: Suhrkamp 1974, S. 20 ff. u. 63 ff.; ders., Das Altern der Moderne, in: Friedeburg/Habermas (Hrsg.), Adorno-Konferenz 1983, a.a.O., S. 177-197; Matei Calinescu, Five Faces of Modernity. Modernism Avant-Garde - Decadence - Kitsch - Postmodernism, Bloomington/London: Indiana University Press 1977, S. 95 ff. u. 265 ff.; Michael T. Jones, Avant-garde: The Convulsions of a Concept, in: Studies in Twentieth Century Literature 5 (1980-81), S. 27-40; Andreas Huyssen, In Search for Tradition. Avantgarde and Postmodernism in the 1970s, in: New German Critique 22 (1981), S. 23-40; Ferenc Fehér, Was ist jenseits von Kunst? Zu den Theorien der Nachmoderne, in: David Roberts (Hrsg.), Tendenzwenden. Aspekte des Kulturwandels der Siebziger Jahre, Frankfurt am Main/Bern/New York: Lang 1984, S. 91-105; Rosalind E. Krauss, The Originality of the Avant-Garde and Other Modernist Myths, Cambridge, Mass./London: MIT Press 1985; Hannes Böhringer, Begriffsfelder. Von der Philosophie zur Kunst, Berlin: Merve 1985, S. 109 ff.; ferner Hermann Lübbe, Im Zug der Zeit. Verkürzter Aufenthalt in der Gegenwart, Berlin/Heidelberg/New York: Springer 1992, S. 91 ff. [39] Jean-François Lyotard, Beantwortung der Frage: Was ist postmodern?, in: Tumult. Zeitschrift für Verkehrs-wissenschaft 4, 1982, S. 131-142 (hier S. 140). Demgegenüber hatte Baudelaire die Modernität der Kunst noch als jenen flüchtigen Augenblick einer prinzipiell vergänglichen Mode beschrieben, in dem sich zugleich eine klassische bzw. "ewige" Bedeutsamkeit im Sinne eines zukünftigen künstlerischen Kanons offenbart. Vgl. Charles Baudelaire, Das Schöne, die Mode und das Glück. Constantin Guys, der Maler des modernen Lebens (frz. 1863), Berlin: Alexander 1988, S. 20 ff.

[40] Zu dieser bei genauerer Betrachtung des Postmodernismus sich geradezu aufdrängenden Schlußfolgerung siehe statt vieler anderer Belege nur die entsprechenden Ausführungen bei Wolfgang Welsch, Die Geburt der postmodernen Philosophie aus dem Geist der modernen Kunst, in: Philosophisches Jahrbuch 97 (1990), S. 15-37; vgl. ferner ders., Unsere postmoderne Moderne, Weinheim: VCH 1987, bes. S. 45 ff. u. 185 ff.

[41] So verstehe ich unter anderem die entsprechenden Ausführungen bei Hermann Lübbe, Im Zug der Zeit, a.a. 
O., S. 91 ff.; ähnlich argumentiert im übrigen auch Luhmann, Die Kunst der Gesellschaft, a.a.O., S. 489 ff. [42] Siehe hierzu auch die entsprechende Schlußfolgerung, zu der Luhmann anläßlich seiner umfassenden Analyse der gesellschaftlichen Ausdifferenzierung des modernen Kunstsystems gekommen ist: "Das Kunstsystem vollzieht Gesellschaft an sich selbst als exemplarischem Fall. Es zeigt, wie es ist. Es zeigt, auf was die Gesellschaft sich eingelassen hatte, als sie Funktionssysteme ausdifferenzierte und sie damit einer autonomen Selbstregulierung überließ. Es zeigt an sich selbst, daß die Zukunft durch die Vergangenheit nicht mehr garantiert ist, sondern unvorhersehbar geworden ist. Operative Schließung, Emanzipation von Kontingenz, Selbstorganisation, Polykontexturalität, Hyperkomplexität der Selbstbeschreibungen oder einfacher und unverständlicher formuliert: Pluralismus, Relativismus, Historismus, all das sind nur verschiedene Anschnitte dieses Strukturschicksals der Moderne. Die Kunst zeigt in der Form des Leidens an sich selbst, daß es so ist, wie es ist. Wer dies wahrnehmen kann, sieht in der modernen Kunst das Paradigma der modernen Gesellschaft." (Die Kunst der Gesellschaft, S. 499).

[43] Vgl. entsprechend Luhmann, Beobachtungen der Moderne, a.a.O., S. 7; ferner ders., Archimedes und wir, hrsg. v. Dirk Baecker u. Georg Stanitzek, Berlin: Merve 1987, S. 165 f.

[44] Siehe hierzu auch Dirk Baecker, Die Kunst der Unterscheidungen, in: Ars Electronica (Hrsg.), Im Netz der Systeme, Berlin: Merve 1990, S. 7-39.

[45] Dies schließt natürlich nicht aus, sondern macht es umgekehrt geradezu erst möglich, daß die rasche Abfolge der mit solcherart von "Zeitdiagnosen" verbundenen Modernitätsdiskurse nun ihrerseits unter genuin historischen Gesichtspunkten analysiert werden kann. Siehe hierzu z.B. die auf die nordamerikanische Intellektuellenszene der letzten fünfzig Jahre bezogene Untersuchung von Jeffrey C. Alexander, Modern, Anti, Post, and Neo: How Social Theories Have Tried to Understand the "New World" of "Our Time", in: Zeitschrift für Soziologie 23 (1994), S. 165-197.

[46] Zu diesem nicht zuletzt mit dem Siegeszug der modernen Kunst verbundenen Verlust der Mitte siehe auch die einschlägige Untersuchung von Hans Sedlmayr, Verlust der Mitte. Die bildende Kunst des 19. und 20. Jahrhunderts als Symptom und Symbol der Zeit, Salzburg: Otto Müller 1948. Daß eine solche "Dezentrierung" aber nicht notwendig zu einer intellektuellen Dauerdepression führen muß, sondern überhaupt erst einen grundlegenden Habitus-Wandel von einer ursprünglich vor lauter Ernst triefenden Intellektualität hin zu einer nun eher südländisch anmutenden Gelassenheit ermöglicht und diese Einstellungsänderung heute gerade auch unter genuin soziologischen Gesichtspunkten gerechtfertigt ist, zeigt nicht zuletzt die entsprechende atmosphärische Einstimmung in solch ein unter bestimmten Voraussetzungen sogar spielerisch leicht zu ertragendes Zeitalter von Ingeborg Heidemann, Der Begriff des Spieles und das ästhetische Weltbild in der Philosophie der Gegenwart, Berlin: de Gruyter 1968.

(C) 2001-2003 Fachbereich Gesellschaftswissenschaften, Johann Wolfgang Goethe-Universität Frankfurt/Main 